\title{
SEMI-RATIONAL SOLUTIONS OF THE THIRD-TYPE DAVEY-STEWARTSON EQUATION
}

\author{
JIGUANG RAO ${ }^{1}$, KUPPUSWAMY PORSEZIAN $^{2}$, JINGSONG HE ${ }^{1}$ \\ ${ }^{1}$ Department of Mathematics, Ningbo University, Ningbo, Zhejiang 315211, P. R. China \\ ${ }^{2}$ Department of Physics, Pondicherry University, Puducherry 605014, India
}

\begin{abstract}
General dark solitons and mixed solutions consisting of dark solitons and breathers for the third-type Davey-Stewartson (DS-III) equation are derived by employing the bilinear method. By introducing the two differential operators, semi-rational solutions consisting of rogue waves, breathers and solitons are generated. These semi-rational solutions are given in terms of determinants whose matrix elements have simple algebraic expressions. Under suitable parametric conditions, we derive general rogue wave solutions expressed in terms of rational functions. It is shown that the fundamental (simplest) rogue waves are line rogue waves. It is also shown that the multi-rogue waves describe interactions of several fundamental rogue waves, which would generate interesting curvy wave patterns. The higher order rogue waves originate from a localized lump and retreat back to it. Several types of hybrid solutions composed of rogue waves, breathers and solitons have also been illustrated. Specifically, these semi-rational solutions have a new phenomenon: lumps form on dark solitons and gradual separation from the dark solitons is observed.
\end{abstract}

Keywords: Davey-Stewartson III equation, Bilinear transformation method, Rogue waves, Semi-rational solutions.

2000 Mathematics Subject Classification: 35Q51, 35Q55 37K10, 37K35, 37K40

PACS numbers: 02.30.Ik, 02.30.Jr, 05.45.Yv

Like one dimensional nonlinear systems, the higher dimensional nonlinear systems are also widely used to explain the dynamics of many higher dimensional physical systems. When compared with one dimensional nonlinear systems, the higher dimensional nonlinear systems are less studied mainly because of the non availability of analytical methods like in one dimension. For instance, during and after 1980s, several higher dimensional systems like the Davey-Stewartson (DS) equation, the Kadomtsev-Petviashvil(KP) equation, Ishimori equation, Three wave equation and so on have been introduced and investigated for Line solitons, Dromions, Lump solutions, vortex solutions etc. For the past one decade or so, the concept of rogue waves have been well reported in many one dimensional nonlinear physical systems but, again, not well studied in higher dimensional systems. The main focus of this paper is to address this problem and investigate rogue wave solutions of one such a higher dimensional system. In this paper, we consider one such a equation in higher dimension, namely the DSIII equation and derive general $N$ th-order dark solitons by reducing the Gram-type solutions of the KP hierarchy. Considering suitable parametric conditions, a new kind of mixed solutions that include breathers, line breathers and dark solitons are given in terms of Gram determinants. Further, general form of semi-rational solutions containing rogue waves, breathers and dark solitons are generated and show that the fundamental

\footnotetext{
* Corresponding author: hejingsong@nbu.edu.cn, jshe@ustc.edu.cn.
} 
rogue waves are actually line rogue waves, which arise from the constant background and retreat back to the constant background again. We also show that the multi-rogue waves demonstrate the interaction of several fundamental line rogue waves, and occurrence of interesting curvy wave patterns due to interaction. The multi-semi-rational solutions possess interesting dynamics, such as more lumps form on multi-dark solitons, such as interaction of lumps, line breathers and dark solitons. A new kind of line rogue waves which are localized both in space and time are illustrated. The higher order semi-rational solutions illustrating the dynamics that more gradual separation of lumps from the one soliton have also been shown, these dynamics correspond to the numerical study of blow up solutions in KP equation.

\section{INTRODUCTION}

For the past one decade or so, the investigation of rogue waves has attracted a lot of attention because of their potential applications in many areas of science and technology. Though initially this identification was done purely from mathematical point of view, over the years, it has been experimentally observed in Ocean technology, Ultra broadband supercontinuum generation, water tank, optical fiber etc. Originally, rogue waves or freak waves are coined for vivid description of the mysterious and monstrous ocean waves. Usually, they are localized and isolated surface waves with large amplitude, which apparently appear from nowhere and disappear without a trace [1 -5]. Subsequently, intensive researches about the characteristics of rogue waves have been implemented in other physical areas, such as Bose-Einstein condensates [6, 7], nonlinear optical system [8 10], superfluids [11], plasma [12,13] even in nonlocal media [14]. In the recent past, some important studies indicated that the rogue waves possess some hallmark phenomenological features including following an unusual $L$-shaped statistics $15-18$. Mathematically, rogue wave solution was first time reported in the nonlinear Schrödinger (NLS) equation by Peregrine [19]. The basic properties of this rogue wave can be summarized as: (i) (quasi-) rational solutions; (ii) localized in both time and space; and (iii) large amplitude (their peak has a height of at least three times of the background) allocated with two hollows. This is considered as the 'definition' of the first-order $(1+1)$ dimensional rogue wave solution. Recently, higher-order rogue waves in the NLS equation were reported in many other articles 20 27]. To date, exact rogue wave solutions have been found in a variety of nonlinear integrable systems, such as three wave interaction equation [28 30], the Sasa-Satsuma equation [31 34], the Hirota equation [35], the derivative nonlinear Schrödinger equation [36], even as some coupled nonlinear Schrödinger system [28, 37, 42], etc [43 48]. In addition to the identification of rogue waves in integrable systems, rogue waves in dissipative dynamical systems has also been reported [17,49].

Though several interesting results are reported for the one dimensional nonlinear equations, to the best of our knowledge, only few results are available in higher dimensional nonlinear systems. This is mainly because of the complications in solving higher dimensional systems and tedious numerical analysis. For example, several higher dimensional nonlinear equations have been derived in ocean, Fluid dynamics, bulk materials and ultrafast optics and also the extension of nonlinear dynamics to a higher spatiotemporal dimensionality is inevitable. Especially, ultrafast optics rogue waves are also higher dimensional, where spatial and temporal degrees of freedom cannot be treated separately. This fact has been established through several theoretical and experimental studies [50 56]. Recently, Klein and Saut obtained a subclass of numerical 
solutions for the Kadomtsev-Petviashvili (KP) equation [57,58, these solutions describe that lumps form at a line soliton and also travel with higher speed than the soliton. In this work, motivated by the above facts, we consider one such a nonlinear higher dimensional problem to explore explicit solutions of $(2+1)$-dimensional soliton equations possessing those unique behaviors.

The third-type of Davey-Stewartson (DSIII) equation is given by

$$
i q_{t}-q_{x x}+q_{y y}-2 \lambda q\left[\left(\int_{-\infty}^{x}|q|_{y}^{2} d x^{\prime}+u_{1}(y, t)\right)-\left(\int_{-\infty}^{y}|q|_{x}^{2} d y^{\prime}+u_{2}(x, t)\right)\right]=0, \lambda= \pm 1,
$$

where the complex valued scalar function $q(x, y, t)$ represents a certain physical quantity and $u_{1}(y, t)$ and $u_{2}(x, t)$ are two arbitrary real-valued functions. Using the transformations

$$
V_{x}=\left(|q|^{2}\right)_{y}, \quad V=\int_{-\infty}^{x}\left(|q|^{2}\right)_{y} d x^{\prime}+u_{1}(y, t)
$$

and

$$
U_{y}=\left(|q|^{2}\right)_{x}, \quad U=\int_{-\infty}^{y}\left(|q|^{2}\right)_{x} d y^{\prime}+u_{2}(x, t),
$$

equation (1) becomes the following system of coupled partial differential equations:

$$
\begin{gathered}
i q_{t}-q_{x x}+q_{y y}-2 \lambda q(V-U)=0, \\
V_{x}=\left(|q|^{2}\right)_{y} \\
U_{y}=\left(|q|^{2}\right)_{x}
\end{gathered}
$$

where $V$ and $U$ are two real functions. These equations were obtained by Boiti et al. [59] by employing ideas [60] of the so-called direct linearisation method of Fokas and Ablowitz [61]. However, as noted by Fokas [62], it was first time derived earlier by Schulman [63] and by Fokas and Santini 64, 65] using the symmetry approach.

As mentioned above, the researches about rogue waves in $(2+1)$-dimensional soliton equations are only few 66 70]. These facts actually motivated us to seek more solutions for the third-type Davey-Stewartson (DSIII) equation. Although Liu et al. have obtained parallel line rogue waves for the DSIII equation [71], but there still exist other kinds of rogue waves, i.e., multi-rogue waves, and higher order rogue waves. Besides, a various forms of hybrid solutions for the DSIII equation have not been studied before, such as solutions consisting of lumps and soltions, such as solutions describing rogue waves interacting with solitons and breathers, and so on.

This paper is organized as follows. In section 2, dark solitons and mixed solutions consisting of dark solitons and breathers of the DSIII equation are given in terms of Gram-type determinants of $N \times N$ matrices. In section 3, rational and semi-rational solutions which include rogue waves, solitons and breathers are expressed in terms of determinant. The dynamical features of rogue waves and semi-rational solutions are demonstrated in section 4 . Our results are summarized in section 5 .

\section{Solitons, Breathers Via Determinants Of $N \times N$ Matrices}

In this section, we derive the explicit form of solutions consisting of solitons and breathers for DSIII equation (3). Using the following dependent variable bilinear transformations

$$
q=\frac{g}{f}, \quad V=-\lambda(\log f)_{y y}, \quad U=-\lambda(\log f)_{x x},
$$


the DSIII equation can be transformed into the following bilinear forms

$$
\begin{aligned}
\left(i D_{t}-D_{x}{ }^{2}+D_{y}{ }^{2}\right) g \cdot f & =0, \\
\left(\lambda D_{x} D_{y}-2\right) f \cdot f & =-2 g \cdot g^{*} .
\end{aligned}
$$

Here, $f$ is a real function, $g$ is a complex function, asterisk denotes complex conjugation, and the operator $D$ is the Hirota's bilinear differential operator [72] defined by

$$
\begin{aligned}
& P\left(D_{x}, D_{y}, D_{t},\right) F(x, y, t \cdots) \cdot G(x, y, t, \cdots) \\
& =\left.P\left(\partial_{x}-\partial_{x^{\prime}}, \partial_{y}-\partial_{y^{\prime}}, \partial_{t}-\partial_{t^{\prime}}, \cdots\right) F(x, y, t, \cdots) G\left(x^{\prime}, y^{\prime}, t^{\prime}, \cdots\right)\right|_{x^{\prime}=x, y^{\prime}=y, t^{\prime}=t},
\end{aligned}
$$

where $P$ is a polynomial of $D_{x}, D_{y}, D_{t}, \cdots$.

Theorem 1. The DSIII equation admits both soliton and breather solutions of the form

$$
q=\frac{g}{f}, \quad V=-\lambda(\log f)_{y y}, \quad U=-\lambda(\log f)_{x x},
$$

where $f$ and $g$ are given by the following Gram determinant

$$
f=\operatorname{det}_{1 \leq i, j \leq N}\left(m_{i, j}^{(0)}\right), g=\operatorname{det}_{1 \leq i, j \leq N}\left(m_{i, j}^{(1)}\right),
$$

and the matrix element $m_{i, j}^{(n)}$ is defined by

$$
m_{i, j}^{(n)}=\gamma_{i j}+\frac{1}{p_{i}+q_{j}}\left(-\frac{p_{i}}{p_{j}^{*}}\right)^{n} \psi_{i} \phi_{j},
$$

with

$$
\begin{aligned}
\psi_{i} & =e^{\xi_{i}}, \phi_{j}=e^{\eta_{j}} \\
\xi_{i} & =\lambda p_{i} x+\frac{1}{p_{i}} y-i\left(\frac{1}{p_{i}^{2}}+p_{i}^{2}\right) t+\xi_{i 0}, \\
\eta_{j} & =\lambda p_{j}^{*} x+\frac{1}{p_{j}^{*}} y+i\left(\frac{1}{p_{j}^{* 2}}+p_{i}^{* 2}\right) t+\xi_{j 0}^{*} .
\end{aligned}
$$

Here $i, j, N$ are arbitrary positive integers, $p_{i}$ and $\xi_{i 0}$ are arbitrary complex constants, $\gamma_{i j}=$ 1,0. Note that a grammian $G=\operatorname{det}_{1 \leq i, j \leq N}\left(g_{i j}\right)$ is the determinant of a matrix with entries $g_{i j}=\int_{a_{0}}^{b_{0}} f_{i} f_{j} d x$

Remark 1. Setting $\gamma_{i j}=1$ when $i=j$, and $\gamma_{i j}=0$ when $i \neq j$, solutions (6) are generting $N$ th-order dark solitons (see Fig.1 (a) and Fig.1(b) for first two dark solitons.). An explicit form of the first order dark soliton is given in eq. (16).

Remark 2. Setting $\gamma_{i j}=1, N \geq 2$, and $p_{i}$ are real parameters, solutions (6) admit the mixed solution of dark solitons and soliton-type solutions (see Fig.1(d)).

Remark 3. Setting $\gamma_{i j}=1, N \geq 2$, and some parameters $p_{i}$ are complex, but $p_{i} \neq p_{j}^{*}(i \neq j)$, solutions (6) are a hybrid of breathers and solitons (see Fig,1(c)).

Remark 4. Setting $\gamma_{i j}=1, N \geq 2$, and some parameters $p_{i}$ are complex and also satisfy $p_{i}=p_{j}^{*}(i \neq j)$, the resulting solutions (6) are a hybrid of line breathers [73], breathers and dark solitons (see Figs.3, 4).

The fact that these solutions are non-singular in nature which can be proved by the same method as for the non-singularity of solutions in Ref [74], thus its detailed proof is omitted here. Next we provide a short proof for this theorem based on the lemma below. 


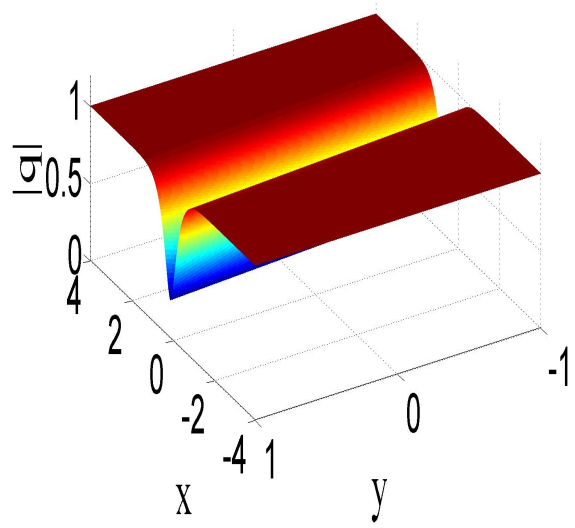

(a)

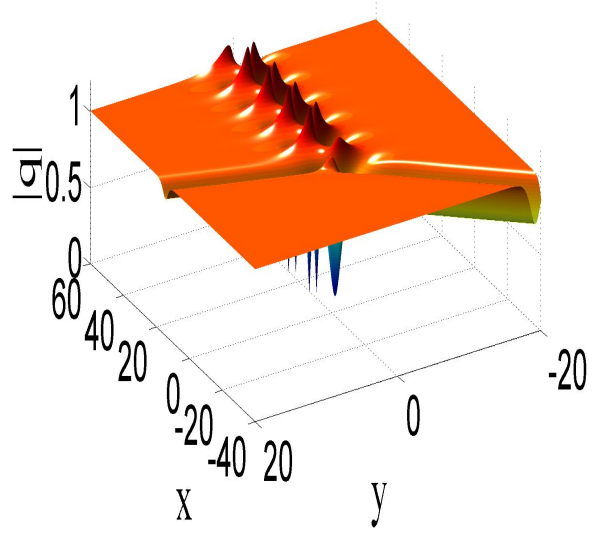

(c)

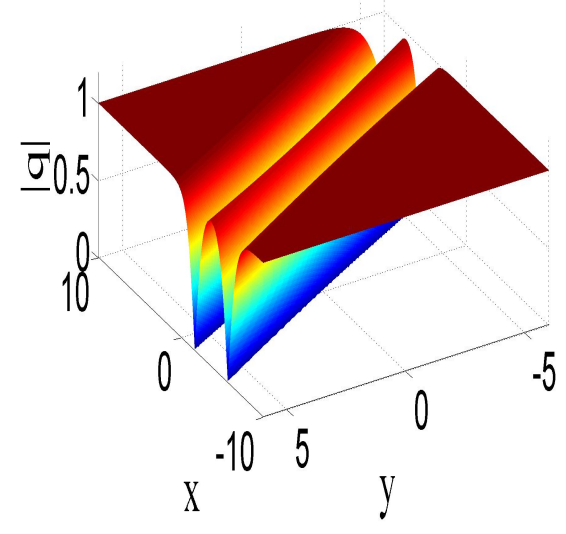

(b)

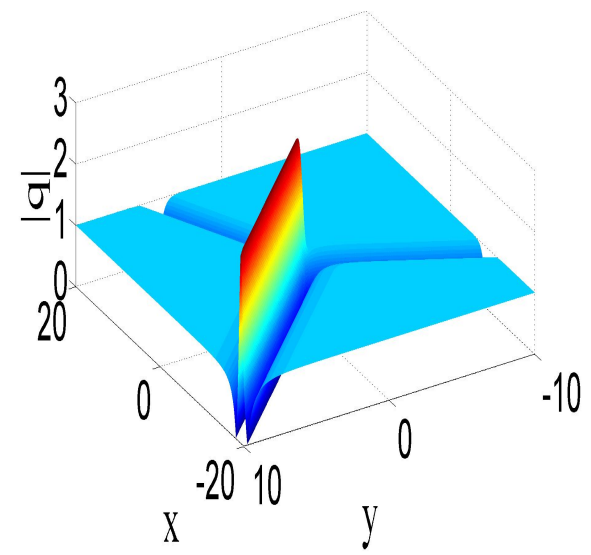

(d)

Figure 1. Dynamics of solutions of the DSIII equation defined in equation (6). (a) One-dark soliton with parameters $N=1, \gamma_{11}=1, \lambda=1, p_{1}=2+i, \xi_{01}=$ $0, t=0$. (b) A second-order dark soliton with parameters $N=2, \gamma_{11}=1, \gamma_{12}=$ $0, \gamma_{21}=0, \gamma_{22}=1, \lambda=1, p_{1}=1, p_{2}=\frac{9}{10}, \xi_{01}=0, \xi_{02}=0, t=0$. (c) A hybrid of dark soliton and a breather with parameters $N=2, \gamma_{i j}=1(i, j=1,2), \lambda=$ $1, p_{1}=\frac{1}{2}+i, p_{2}=\frac{1}{2}+\frac{1}{2} i, \xi_{01}=0, \xi_{02}=0, t=0$. (d) A hybrid of a dark soliton and a soliton-type solution with parameters $N=2, \gamma_{i j}=1(i, j=1,2), \lambda=$ $1, p_{1}=\frac{1}{2}, p_{2}=1, \xi_{01}=0, \xi_{02}=0, t=0$.

Lemma 2.1. Let $m_{i j}^{(n)}, \psi_{i}^{(n)}$ and $\phi_{j}^{(n)}$ be functions of variables $x_{-1}, x_{-2}, x_{1}, x_{2}$ satisfying the following differential and difference relations,

$$
\begin{aligned}
\partial_{x_{1}} m_{i j}^{(n)} & =\psi_{i}^{(n)} \phi_{j}^{(n)} \\
\partial_{x_{-1}} m_{i j}^{(n)} & =-\psi_{i}^{(n-1)} \phi_{j}^{(n+1)} \\
\partial_{x_{2}} m_{i j}^{(n)} & =\psi_{i}^{(n+1)} \phi_{j}^{(n)}+\psi_{i}^{(n)} \phi_{j}^{(n-1)} \\
\partial_{x_{-2}} m_{i j}^{(n)} & =-\psi_{i}^{(n-2)} \phi_{j}^{(n+1)}-\psi_{i}^{(n-1)} \phi_{j}^{(n+2)}, \\
\partial_{x_{v}} \psi_{i} & =\psi_{i}^{(n+v)}, \\
\partial_{x_{v}} \phi_{j} & =-\phi_{j}^{(n-v)} 5(v=-2,-1,1,2) .
\end{aligned}
$$




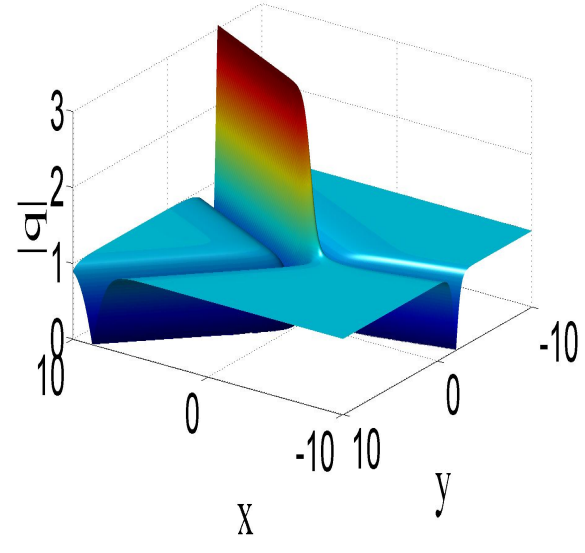

(a) $t=-10^{4}$

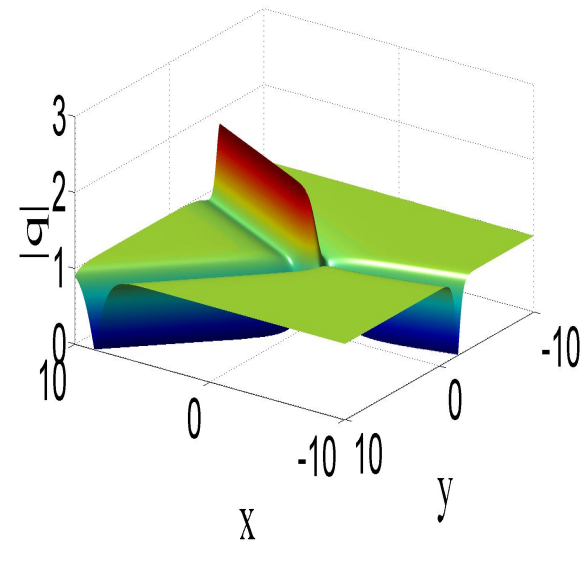

(c) $t=10$

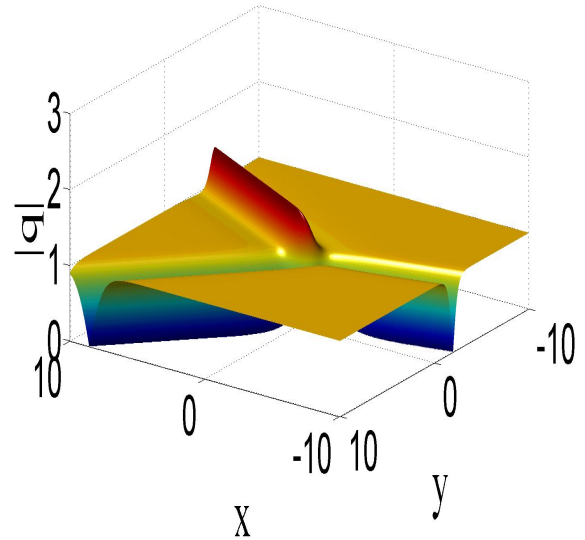

(b) $t=-10$

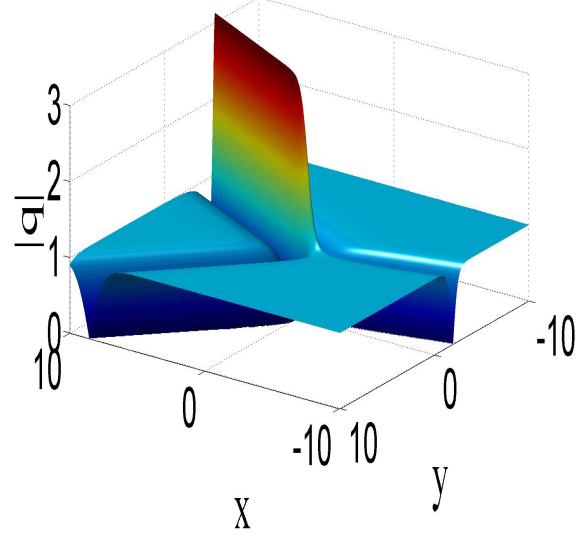

(d) $t=10^{4}$

FigURE 2. The time evolution of mixed solutions consisting of a dark soliton and soliton-type solution of the DSIII equation given by equation (6) with parameters $N=2, \gamma_{i j}=1(i, j=1,2), p_{1}=\frac{1}{2}, p_{2}=1, \lambda=-1, \xi_{01}=0, \xi_{02}=0$.

Then the determinant

$$
\tau_{n}=\operatorname{det}_{1 \leq i, j \leq N}\left(m_{i j}^{(n)}\right)
$$

satisfies the following bilinear equations in $K P$ hierarchy

$$
\begin{aligned}
\left(D_{x_{1}}^{2}-D_{x_{2}}\right) \tau_{n+1} \cdot \tau_{n} & =0 \\
\left(D_{x_{-1}}^{2}+D_{x_{-2}}\right) \tau_{n+1} \cdot \tau_{n} & =0 \\
\left(D_{x_{1}} D_{x_{-1}}-2\right) \tau_{n} \cdot \tau_{n} & =-2 \tau_{n+1} \tau_{n-1} .
\end{aligned}
$$

This Lemma can be proved by a similar way as the proof of the Lemma 1 in Ref. [74] or the proof of the Lemma 3.2 in Ref [26], thus we omit its proof in this paper. Next we provide a short proof of Theorem 1 based on this Lemma. 


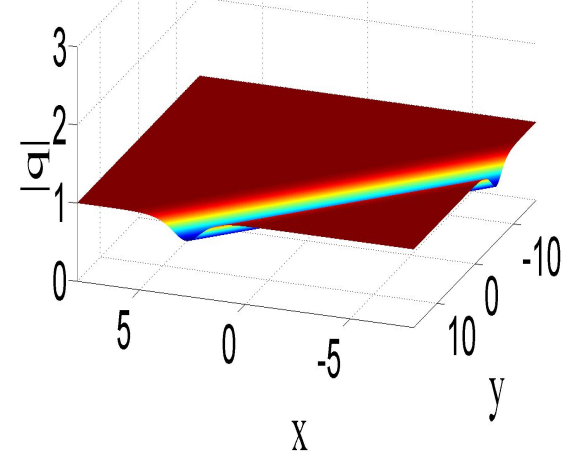

(a) $t=-5$

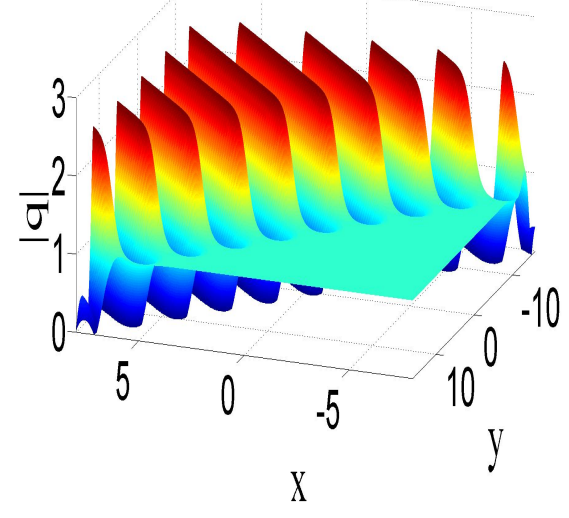

(c) $t=0$

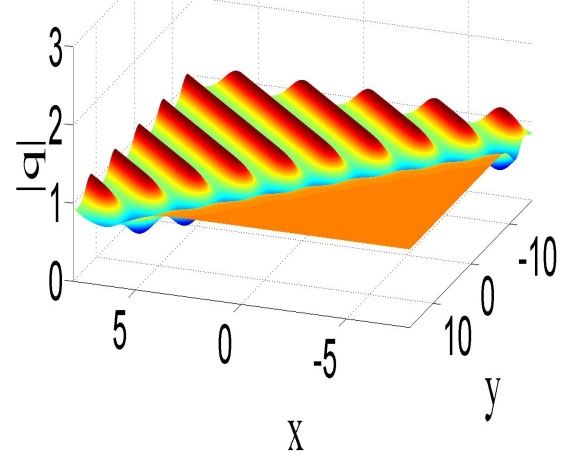

(b) $t=-1$

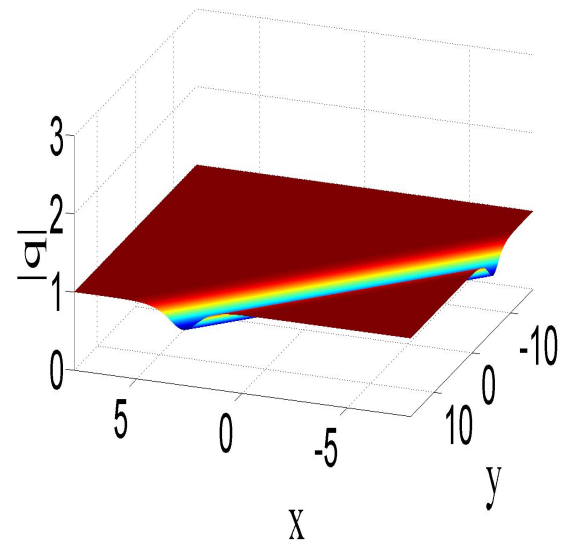

(d) $t=5$

Figure 3. The time evolution of mixed solutions consisting of a dark soliton and periodic line wave of the DSIII equation given by equation (6) with parameters $N=2, \gamma_{i j}=1(i, j=1,2), p_{1}=1+i, p_{2}=1-i, \lambda=-1, \xi_{01}=0, \xi_{02}=0$.

Proof of Theorem 1. In order to prove Theorem 1, functions $m_{i j}^{(n)}, \psi_{i}^{(n)}$ and $\phi_{j}^{(n)}$ are selected by the following forms

$$
\begin{aligned}
m_{i j}^{(n)} & =\gamma_{i j}+\frac{1}{p_{i}+q_{j}} \psi_{i}^{(n)} \phi_{j}^{(n)} \\
\psi_{i}^{(n)} & =p_{i}^{n} e^{\xi_{i}} \\
\phi_{j}^{(n)} & =\left(-q_{j}\right)^{-n} e^{\eta_{j}}
\end{aligned}
$$

where $\xi_{i}=\frac{1}{p_{i}^{2}} x_{-2}+\frac{1}{p_{i}} x_{-1}+p_{i} x_{1}+p_{i}^{2} x_{2}+\xi_{i 0}, \eta_{j}=-\frac{1}{q_{j}^{2}} x_{-2}+\frac{1}{q_{j}} x_{-1}+q_{j} x_{1}-q_{j}^{2} x_{2}+\eta_{j 0}$, and $p_{i}, q_{j}, \xi_{i 0}, \eta_{j 0}$ are arbitrary complex constants.

Furthermore, taking the parametric condition

$$
q_{j}=p_{j}^{*}, \quad \eta_{j 0}=\xi_{i 0}^{*},
$$




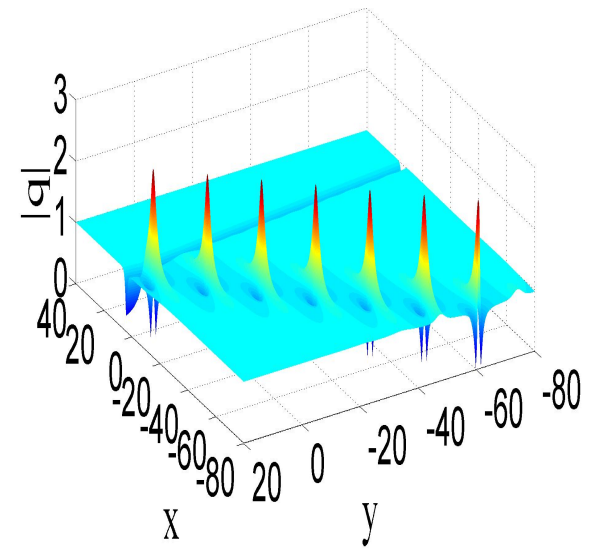

(a) $t=-10$

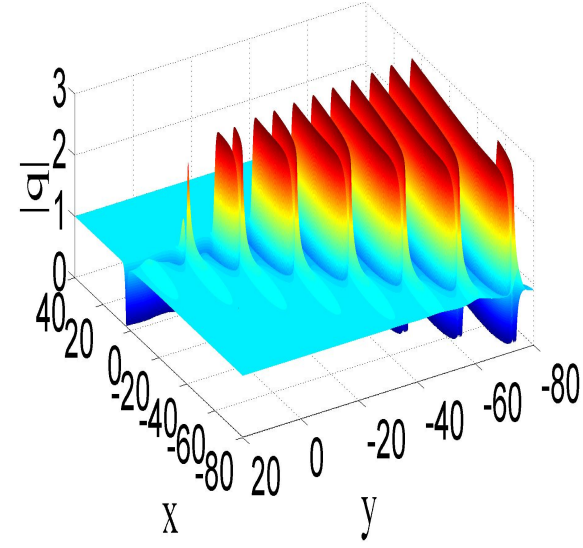

(c) $t=0$

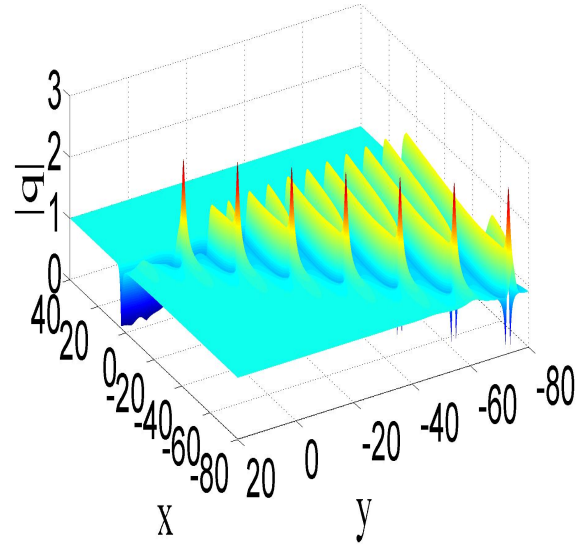

(b) $t=-1$

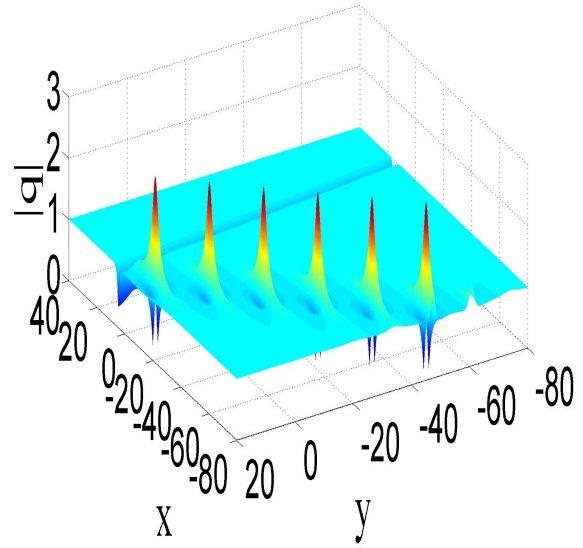

(d) $t=10$

FigURE 4. The time evolution of mixed solutions consisting of a breather, a dark soliton and periodic line waves of the DSIII equation given by equation (6) with parameters $N=3, \gamma_{i j}=1(i, j=1,2,3), p_{1}=1-\frac{i}{2}, p_{2}=1+\frac{i}{2}, p_{3}=1, \lambda=$ $1, \xi_{01}=0, \xi_{02}=0, \xi_{03}=0$.

and assuming $x_{-1}, x_{1}$ are real, $x_{-2}, x_{2}$ are pure imaginary, we have

$$
\eta_{j}^{\prime}=\xi_{j}^{*}, \quad m_{i j}^{*}(n)=m_{j i}(-n), \quad \tau_{n}^{*}=\tau_{-n} .
$$

Applying the change of independent variables $x_{-2}=-i t, x_{-1}=y, x_{1}=\lambda x, x_{2}=-i t$, and setting $\tau_{0}=f, \tau_{1}=g, \tau_{-1}=g^{*}$, then the bilinear equation (12) can be transformed into the bilinear equation (5). Thus solutions of DSIII equation (3) expressed in Theorem 1 can be generated from solutions of bilinear equation (12). Hence the Theorem 1 has been proved.

To get the single soliton solution $q$ of the DSIII equation, we take $N=1$ in formula (7). After simple algebra, single soliton solution $q$ can be expressed as

$$
q=\frac{1}{2}\left[1+y_{1}+\left(y_{1}-1\right) \tanh \left(\frac{\xi_{1}+\xi_{1}^{*}+\rho_{1}}{2}\right)\right],
$$


where

$$
\xi_{1}=\lambda p_{1} x+\frac{y}{p_{1}}-i\left(\frac{1}{p_{1}^{2}}+p_{1}^{2}\right) t+\xi_{i 0}, y_{1}=-\frac{p_{1}}{p_{1}^{*}}, e^{\rho_{1}}=\frac{1}{p_{1}+p_{1}^{*}} .
$$

It is easy to see that $|q|$ approaches to the constant background amplitudes 1 as $x, y \rightarrow \pm \infty$, see Fig. 11(a). Besides, at any fixed time, as $x$ varies from $-\infty$ to $+\infty$, the phases of $|q|$ acquires shifts the amount of $2 \phi$, where

$$
y_{1}=e^{2 i \phi} .
$$

At the center of the soliton where $\xi_{1}+\xi_{1}^{*}+\rho_{1}=0$, intensity of the single soliton is

$$
|q|_{\text {center }}=\cos \phi<1 \text {, }
$$

here the constant amplitude is 1 . Thus the center intensity is lower than the constant background intensity, which means the single soliton is a dark soliton.

In addition to dark-dark soliton solutions, these solutions also possess other dynamical behaviours. To analysis the typical behaviours of these mixed solutions, we consider the case of $N=2, \gamma_{i j}=1(i, j=1,2)$. In this case, the corresponding solution can be classify into three case:

case 1: $p_{1}, p_{2}$ are real.

In this case, the corresponding solution is a mixture of dark soliton and soliton-type solution. This solution with parameters

$$
p_{1}=\frac{1}{2}, p_{2}=1, \lambda=-1, \xi_{10}=\xi_{20}=0,
$$

is expressed as

$$
q=\frac{-\mathrm{e}^{4 y}-24 \mathrm{e}^{\frac{9}{4} i t+y+\frac{3}{2} x}-6 \mathrm{e}^{-\frac{9}{4} i t+y+\frac{3}{2} x}+18 \mathrm{e}^{2 x+2 y}+9 \mathrm{e}^{x}}{24 \mathrm{e}^{y+\frac{3}{2} x} \cos \left(\frac{9}{4} t\right)-18 \mathrm{e}^{2 x+2 y}-\mathrm{e}^{4 y}-9 \mathrm{e}^{x}} .
$$

The time evolution of this solution is shown in Fig. 2. It is seen that this solution is composed of a dark soliton and a soliton-type solution. The soliton-type solution behaves as a $W$-shaped soliton and ends on the dark soliton. However, different from the soliton solution, this solitontype solution possesses altering amplitudes.

case 2: $p_{1}, p_{2}$ are complex, and $p_{1}^{*} \neq p_{2}$.

The corresponding solutions consist of a dark soliton and a breather, and the breather ends on the dark soliton. This solution with parameters

$$
p_{1}=\frac{1}{2}+i, p_{2}=\frac{1}{2}+\frac{1}{2} i, \lambda=-1, \xi_{10}=\xi_{20}=0,
$$

is expressed as

where

$$
q=\frac{g}{f}
$$

$$
\begin{aligned}
f= & 5 \mathrm{e}^{-3 t-x+2 y}+\mathrm{e}^{-\frac{57}{25} t+\frac{14 y}{5}-2 x}+5 \mathrm{e}^{\frac{18}{25} t+\frac{4}{5} y-x}-(4-2 i) \mathrm{e}^{-\frac{57}{50} t+\frac{7}{5} y+\frac{i}{5} y-x-\frac{i}{2} x+\frac{123 i}{100} t} \\
& -(4+2 i) \mathrm{e}^{-\frac{57}{50} t-\frac{123 i}{100} t+\frac{7}{5} y-\frac{i}{5} y-x+\frac{i}{2} x}, \\
g= & -5 i \mathrm{e}^{-3 t-x+2 y}-\left(\frac{4}{5}+\frac{3}{5} i\right) \mathrm{e}^{-\frac{57}{25} t+\frac{14 y}{5}-2 x}+(3-4 i) \mathrm{e}^{\frac{18}{25} t+\frac{4}{5} y-x}+ \\
& (1+7 i) \mathrm{e}^{-\frac{57 t}{50}+\frac{7}{5} y+\frac{i}{5} y-x-\frac{i}{2} x+\frac{123 i}{100} t}-(2-2 i) \mathrm{e}^{-\frac{57}{50} t-\frac{123 i}{100} t+\frac{7}{5} y-\frac{i}{5} y-x+\frac{i}{2} x} .
\end{aligned}
$$

This solution is shown in Fig. 1 (c). 
case 3: $p_{1}, p_{2}$ are complex, and $p_{1}^{*}=p_{2}$.

The corresponding solutions consist of a dark soliton and a line breather, and the line breather also ends on the dark soliton. This solution with parameters

$$
p_{1}=1+i, p_{2}=1-i, \lambda=-1, \xi_{10}=\xi_{20}=0,
$$

is expressed as

$$
q=\frac{-(-2+2 i) \mathrm{e}^{-i(2 x+y)}+(2+2 i) \mathrm{e}^{i(2 x+y)}+\mathrm{e}^{-2 x+y}+4 i\left(\mathrm{e}^{-3 t}-\mathrm{e}^{3 t}\right)}{(-2+2 i) \mathrm{e}^{-i(2 x+y)}-(2+2 i) \mathrm{e}^{i(2 x+y)}+\mathrm{e}^{-2 x+y}+4\left(\mathrm{e}^{-3 t}+\mathrm{e}^{3 t}\right)} .
$$

This solution is shown in Fig. 3. As seen that, this solution is a dark soliton when $t \rightarrow \pm \infty$. In the intermediate time, periodic line waves arise from the constant background and then decay back to the same background (see the panels at $t=-1,0$ ). As line rogue wave can be treated as a limited case of these periodic line wave, hereafter we refer to these periodic line waves as line breather $[73]$.

For larger $N$ and different choices of $p_{j}$, these solutions given by equation (6) possess various dynamical behaviours, such as a hybrid of multi-breathers and multi-dark solitons, a hybrid of usual breathers, line breathers and multi-dark solitons. Fig. 4 demonstrates one type of these mixed solutions. As can be seen, this solution is only the combination of a dark soliton and a breather when $t \rightarrow-\infty$ ( see the $t=-10$ panel ). In the intermediate time, a line breather arises from the constant background, thus the solution is made up of one breather, one line breather and a dark soliton (see the $t=0$ panel). At larger time, the line breather disappears again to this background, and the solution is again mixing of a usual breather and a dark soliton (see the $t=10$ panel). Specifically, the line breather just exists on the region where the dark soliton and the breather cross over each other within a short period of time.

The above discussions just focus on solutions generated by exponential functions, next, we consider the construction of rational and semi-rational solutions of the DSIII equation.

\section{RATiOnAL AND SEMI-RATIONAL SOLUTIONS IN THE DETERMinANT FORM}

Inspired by the works of Y. Ohta and J. Yang [67,68], we construct rational and semi-rational solutions for the DSIII equation by introducing the following differential operators

$$
A_{i}=\sum_{k=0}^{n_{i}} c_{i k}\left(p_{i} \partial_{p_{i}}\right)^{n_{i}-k}, \quad B_{j}=\sum_{l=0}^{n_{j}} c_{j l}^{*}\left(p_{j}^{*} \partial_{p_{j}^{*}}\right)^{n_{j}-l}
$$

where $c_{i k}, c_{j l}$ are arbitrary complex constants. Besides, we define functions $M_{i j}^{(n)}, \Psi_{i}^{(n)}, \Phi_{j}^{(n)}$ as

$$
\begin{aligned}
M_{i j}^{(n)} & =A_{i} B_{j} m_{i, j}^{(n)}, \\
\Psi_{i}^{(n)} & =A_{i} \psi_{i}^{(n)}, \\
\Phi_{j}^{(n)} & =B_{j} \phi_{j}^{(n)},
\end{aligned}
$$


then it is easy to find that $M_{i j}^{(n)}, \Psi_{i}^{(n)}, \Phi_{j}^{(n)}$ also satisfy the differential and difference relations defined in (10),

$$
\begin{aligned}
\partial_{x_{1}} M_{i j}^{(n)} & =\Psi_{i}^{(n)} \Phi_{j}^{(n)}, \\
\partial_{x_{-1}} M_{i j}^{(n)} & =-\Psi_{i}^{(n-1)} \Phi_{j}^{(n+1)}, \\
\partial_{x_{2}} M_{i j}^{(n)} & =\Psi_{i}^{(n+1)} \Phi_{j}^{(n)}+\Psi_{i}^{(n)} \Phi_{j}^{(n-1)}, \\
\partial_{x_{-2}} M_{i j}^{(n)} & =-\Psi_{i}^{(n-2)} \Phi_{j}^{(n+1)}-\Psi_{i}^{(n-1)} \Phi_{j}^{(n+2)}, \\
\partial_{x_{v}} \Psi_{i} & =\Psi_{i}^{(n+v)}, \\
\partial_{x_{v}} \Phi_{j} & =-\Phi_{j}^{(n-v)} \quad(v=-2,-1,1,2) .
\end{aligned}
$$

Thus the solutions

$$
f=\operatorname{det}_{1 \leq i, j \leq N}\left(M_{i, j}^{(0)}\right), g=\operatorname{det}_{1 \leq i, j \leq N}\left(M_{i, j}^{(1)}\right),
$$

still satisfy bilinear equation (5).

Through the operator relations

$$
\begin{aligned}
A_{i} p_{i}^{n} e^{\xi_{i}} & =\sum_{k=0}^{n_{i}} c_{i k}\left(p_{i} \partial_{p_{i}}\right)^{n_{i}-k} p_{i}^{n} e^{\xi_{i}}=p_{i}^{n} e^{\xi_{i}} \sum_{k=0}^{n_{i}} c_{i k}\left(p_{i} \partial_{p_{i}}+\xi_{i}^{\prime}+n\right)^{n_{i}-k} \\
B_{j}\left(-p_{j}\right)^{-n} e^{\xi_{j}} & =\sum_{l=0}^{n_{j}} c_{j l}^{*}\left(p_{j}^{*} \partial_{p_{j}^{*}}\right)^{n_{j}-l}\left(-p_{j}^{*}\right)^{-n} e^{\xi_{j}^{*}}=\left(-p_{j}^{*}\right)^{-n} e^{\xi_{j}^{*}} \sum_{l=0}^{n_{j}} c_{j l}^{*}\left(p_{j}^{*} \partial_{p_{j}^{*}}+\xi_{j}^{*}-n\right)^{n_{j}-l},
\end{aligned}
$$

where

$$
\xi_{i}^{\prime}=\lambda p_{i} x-\frac{1}{p_{i}} y-2 i\left(\frac{1}{p i^{2}}-p_{i}^{2}\right) t,
$$

and $\xi_{i}$ are given by equation (9).

The matrix element $M_{i j}$ defined in equation (26) becomes

$$
\begin{aligned}
M_{i j}^{(n)}= & A_{i} B_{j} m_{i j}^{(n)} \\
= & \left(-\frac{p_{i}}{p_{j}^{*}}\right)^{n} e^{\xi_{i}+\xi_{j}^{*}} \sum_{k=0}^{n_{i}} c_{i k}\left(p_{i} \partial_{p_{i}}+\xi_{i}^{\prime}+n\right)^{n_{i}-k} \\
& \times \sum_{l=0}^{n_{j}} c_{j l}^{*}\left(p_{j}^{*} \partial_{p_{j}^{*}}+\xi_{j}^{*}-n\right)^{n_{j}-l} \frac{1}{p_{i}+p_{j}^{*}}+\gamma_{i j} c_{i n_{i}} d_{j n_{j}},
\end{aligned}
$$

hence the semi-rational solutions of bilinear equation (5) are obtained. Taking $\delta_{i j}=0$ and by the gauge freedom of $f$ and $g$, the rational solutions are generated.

Based on the above results, semi-rational solutions of the DSIII equation can be proposed in the following Theorem.

Theorem 2. The DSIII equation (3) has rational and semi-rational solutions of the form

$$
q=\frac{g}{f}, \quad V=-\lambda(\log f)_{y y}, \quad U=-\lambda(\log f)_{x x}
$$

with

$$
f=\operatorname{det}_{1 \leq i, j \leq N}\left(M_{i, j}^{(0)}\right), g=\operatorname{det}_{1 \leq i, j \leq N}\left(M_{i, j}^{(1)}\right)
$$


and the matrix elements in $f$ and $g$ are defined by

$$
\begin{aligned}
M_{i j}^{(n)}= & \left(-\frac{p_{i}}{p_{j}^{*}}\right)^{n} e^{\xi_{i}+\xi_{j}^{*}} \sum_{k=0}^{n_{i}} c_{i k}\left(p_{i} \partial_{p_{i}}+\xi_{i}^{\prime}+n\right)^{n_{i}-k} \\
& \times \sum_{l=0}^{n_{j}} c_{j l}^{*}\left(p_{j}^{*} \partial_{p_{j}^{*}}+\xi_{j}^{*}-n\right)^{n_{j}-l} \frac{1}{p_{i}+p_{j}^{*}}+\gamma_{i j} c_{i n_{i}} c_{j n_{j}}^{*},
\end{aligned}
$$

and $\xi_{i}, \xi_{i}^{\prime}$ are given by equations (9) and (28), and $i, j, n_{i}, n_{j}$ are arbitrary positive integers, $c_{i k}, c_{j l}, p_{i}, p_{j}$ are arbitrary complex constants, and $\gamma_{i j}$ are arbitrary real constants.

Remark 5. Setting $\gamma_{i j}=0$ in equation (32) and by the gauge freedom of $f$ and $g$ in equation (30), then the semi-rational solutions (30) become rational solutions for the DSIII equation. These rational solutions can be classified into two types: rogue waves and lumps.

Remark 6. The parameters $\gamma_{i j}$ play a key role in the combination of semi-rational solutions (30). When $\gamma_{i i}=1, \gamma_{i j}=0(i \neq j)$, the obtained semi-rational solutions consist of rogue waves and dark solitons or lumps and dark solitons. When $\gamma_{i j}=1$, the semi-rational solutions are a combination of rogue waves, breathers and dark solitons.

Remark 7. By a scaling of $M_{i j}$ given by equation (32), we can normalize $c_{i 0}=1$ without loss of generality, and thus hereafter we set $c_{i 0}=1$.

Remark 8. For any non-zero column vector $\mu=\left(\mu_{1}, \mu_{2} \ldots \mu_{N}\right)^{T}$ and $\bar{\mu}$ being its complex transpose, we have

$$
\begin{aligned}
\bar{\mu} f \mu= & \sum_{i, j=1}^{N} \overline{\mu_{i}} M_{i, j}^{(0)} \mu_{j} \geq\left.\sum_{i, j=1}^{N} \overline{\mu_{i}} \mu_{j} A_{i} B_{j} \frac{1}{p_{i}+q_{j}} e^{\xi_{i}+\eta_{j}}\right|_{q_{j}=p_{j}^{*}} \\
= & \left.\sum_{i, j=1}^{N} \overline{\mu_{i}} \mu_{j} A_{i} B_{j} \int_{-\infty}^{x} e^{\xi_{i}+\eta_{j}} d x\right|_{q_{j}=p_{j}^{*}} \\
= & \int_{-\infty}^{x}\left(\left.\sum_{i, j=1}^{N} \overline{\mu_{i}} \mu_{j} A_{i} B_{j} e^{\xi_{i}+\eta_{j}}\right|_{q_{j}=p_{j}^{*}}\right) d x \\
= & \int_{-\infty}^{x}\left|\sum_{i=1}^{N} \overline{\mu_{i}} A_{i} e^{\xi_{i}}\right|^{2} d x>0
\end{aligned}
$$

thus we have proved that $f$ is a positive definitive. Therefore, the solutions $q, U$, and $V$ given in this Theorem are non-singular in nature.

The above obtained semi-rational solutions possess various other dynamical behaviours which have never been reported before, such as interactions between rogue waves and dark solitons, interactions between lumps, breathers and dark solitons, and so on. In the section 4, we concentrate on demonstrating these unique dynamics and properties.

\section{Dynamics of Rogue Waves}

In this section, we analyse the dynamics of rational and semi-rational solutions of the DSIII equation, and also illustrate the dynamics of rogue waves. We first consider the fundamental (simplest) semi-rational solutions. 


\subsection{Dynamics of the fundamental semirational solutions.}

The fundamental semi-rational solutions of the DSIII equation are generated from equation (30) by taking $N=1, n_{i}=1$. In this case, they are expressed explicitly by the following formulae :

$$
q=\frac{g}{f}, \quad V=-\lambda(\log f)_{y y}, \quad U=-\lambda(\log f)_{x x},
$$

where

$$
\begin{array}{r}
f=e^{\xi_{1}+\xi_{1}^{*}}\left(\sum_{k=0}^{1} c_{1 k}\left(p_{1} \partial_{p_{1}}+\xi_{1}^{\prime}\right)^{1-k} \sum_{l=0}^{1} c_{1 l}^{*}\left(p_{1}^{*} \partial_{p_{1}^{*}}+\xi_{1}^{\prime *}\right)^{1-l}\right) \frac{1}{p_{1}+p_{1}^{*}}+\gamma_{11} c_{11} c_{11}^{*} \\
=e^{\xi_{1}+\xi_{1}^{*}}\left(p_{1} \partial_{p_{1}}+\xi_{1}^{\prime}+c_{11}\right)\left(p_{1}^{*} \partial_{p_{1}^{*}}+\xi_{1}^{\prime *}+c_{11}^{*}\right) \frac{1}{p_{1}+p_{1}^{*}}+\gamma_{11} c_{11} c_{11}^{*} \\
=\frac{e^{\xi_{1}+\xi_{1}^{*}}}{p_{1}+p_{1}^{*}}\left[\left(\xi_{1}^{\prime}-\frac{p_{1}}{p_{1}+p_{1}^{*}}+c_{11}\right)\left(\xi_{1}^{\prime *}-\frac{p_{1}^{*}}{p_{1}+p_{1}^{*}}+c_{11}^{*}\right)+\frac{p_{1} p_{1}^{*}}{\left(p_{1}+p_{1}^{*}\right)^{2}}\right]+\gamma_{11} c_{11} c_{11}^{*}, \\
g=e^{\xi_{1}+\xi_{1}^{*}}\left(\sum_{k=0}^{1} c_{1 k}\left(p_{1} \partial_{p_{1}}+\xi_{1}^{\prime}+1\right) \sum_{l=0}^{1} c_{1 l}^{*}\left(p_{1}^{*} \partial_{p_{1}^{*}}+\xi_{1}^{*}-1\right) \frac{1}{p_{1}+p_{1}^{*}}+\gamma_{11} c_{11} c_{11}^{*}\right. \\
=e^{\xi_{1}+\xi_{1}^{*}}\left(p_{1} \partial_{p_{1}}+\xi_{1}^{\prime}+c_{11}+1\right)\left(p_{1}^{*} \partial_{p_{1}^{*}}+\xi_{1}^{\prime *}+c_{11}^{*}-1\right) \frac{1}{p_{1}+p_{1}^{*}}+\gamma_{11} c_{11} c_{11}^{*} \\
=\frac{e^{\xi_{1}+\xi_{1}^{*}}}{p_{1}+p_{1}^{*}}\left[\left(\xi_{1}^{\prime}-\frac{p_{1}}{p_{1}+p_{1}^{*}}+c_{11}+1\right)\left(\xi_{1}^{\prime *}-\frac{p_{1}^{*}}{p_{1}+p_{1}^{*}}+c_{11}^{*}-1\right)+\frac{p_{1} p_{1}^{*}}{\left(p_{1}+p_{1}^{*}\right)^{2}}\right]+\gamma_{11} c_{11} c_{11}^{*},
\end{array}
$$

and

$$
\begin{aligned}
& \xi_{1}=\lambda p_{1} x+\frac{1}{p_{1}} y-i\left(\frac{1}{p_{1}^{2}}+p_{1}^{2}\right) t+\xi_{10}, \\
& \xi_{i}^{\prime}=\lambda p_{1} x-\frac{1}{p_{1}} y-2 i\left(\frac{1}{p 1^{2}}-p_{1}^{2}\right) t
\end{aligned}
$$

$p_{1}, \xi_{10}$ and $c_{11}$ are arbitrary complex parameters. Without loss of generality, we set $p_{1}=$ $p_{1 R}+i p_{1 I}, c_{11}=c_{1 R}+i c_{1 I}$ and then rewrite the above functions $f$ and $g$ as

$$
\begin{aligned}
& f=\frac{e^{\xi_{1}+\xi_{1}^{*}}}{2 p_{1 R}}\left(\theta \theta^{*}+\theta_{0}\right)+\gamma_{11}\left(c_{1 R}^{2}+c_{1 I}^{2}\right), \\
& g=\frac{e^{\xi_{1}+\xi_{1}^{*}}}{2 p_{1 R}}\left[(\theta+1)\left(\theta^{*}-1\right)+\theta_{0}\right]+\gamma_{11}\left(c_{1 R}^{2}+c_{1 I}^{2}\right),
\end{aligned}
$$

where

$$
\begin{gathered}
\theta=l_{1}+i l_{2}, \theta_{0}=\frac{p_{1 R}^{2}+p_{1 I}^{2}}{4 p_{1 R}^{2}}, \\
l_{1}=\lambda p_{1 R} x+\frac{p_{1 R}}{p_{1 R}^{2}+p_{1 I}^{2}} y-\left(\frac{4 p_{1 R} p_{1 I}}{\left(p_{1 R}^{2}+p_{1 I}^{2}\right)^{2}}+2 p_{1 R} p_{1 I}\right) t+c_{1 R}+\frac{1}{2}, \\
l_{2}=\lambda p_{1 I} x-\frac{p_{1 I}}{p_{1 R}^{2}+p_{1 I}^{2}} y-\left[\frac{2\left(p_{1 R}^{2}-p_{1 I}^{2}\right)}{\left(p_{1 R}^{2}+p_{1 I}^{2}\right)^{2}}-2\left(p_{1 R}^{2}-p_{1 I}^{2}\right)\right] t+c_{1 I}-\frac{p_{1 I}}{2 p_{1 R}} .
\end{gathered}
$$


Thus the final expression of the semi-rational solutions is obtained as

$$
\begin{gathered}
q=1-\frac{2 i l_{2}+1}{l_{1}^{2}+l_{2}^{2}+\theta_{0}+2 p_{1 R} \gamma_{11}\left(c_{1 R}^{2}+c_{1 I}^{2}\right) e^{-\xi_{1}-\xi_{1}^{*}}}, \\
V=-\lambda \frac{4 p_{1 R} \delta_{11}\left(c_{1 R}^{2}+c_{1 I}^{2}\right) e^{-\xi_{1}-\xi_{1}^{*}} A+B}{\left[l_{1}^{2}+l_{2}^{2}+\theta_{0}+2 p_{1 R} \gamma_{11}\left(c_{1 R}^{2}+c_{1 I}^{2}\right) e^{\left.-\xi_{1}-\xi_{1}^{*}\right]^{2}}\right.}, \\
U=-\lambda \frac{4 p_{1 R} \delta_{11}\left(c_{1 R}^{2}+c_{1 I}^{2}\right) e^{-\xi_{1}-\xi_{1}^{*}} C+D}{\left[l_{1}^{2}+l_{2}^{2}+\theta_{0}+2 p_{1 R} \gamma_{11}\left(c_{1 R}^{2}+c_{1 I}^{2}\right) e^{-\xi_{1}-\xi_{1}^{*}}\right]^{2}},
\end{gathered}
$$

where

$$
\begin{aligned}
A & =\frac{4 p_{1 R}^{2}}{\left(p_{1 R}^{2}+p_{1 I}^{2}\right)^{2}}\left(2 l_{1}^{2}+2 l_{2}^{2}+2 \theta_{0}-4 l_{1}+1\right)+\frac{4 p_{1 R} p_{1 I}}{\left(p_{1 R}^{2}+p_{1 I}^{2}\right)^{2}}+\frac{p_{1 I}^{2}}{\left(p_{1 R}^{2}+p_{1 I}^{2}\right)^{2}}, \\
B & =\frac{2 p_{1 R}^{2}}{\left(p_{1 R}^{2}+p_{1 I}^{2}\right)^{2}}\left(-l_{1}^{2}+l_{2}^{2}+\theta_{0}\right)-\frac{8 p_{1 R} p_{1 I}}{\left(p_{1 R}^{2}+p_{1 I}^{2}\right)^{2}} l_{1} l_{2}+\frac{2 p_{1 I}^{2}}{\left(p_{1 R}^{2}+p_{1 I}^{2}\right)^{2}}\left(l_{1}^{2}-l_{2}^{2}+\theta_{0}\right), \\
C & =\left(2 l_{1}^{2}+2 l_{2}^{2}+2 \theta_{0}+4 \lambda_{1} l_{1}+1\right) p_{1 R}^{2}+4 \lambda p_{1 R} p_{1 I} l_{2}+2 \lambda^{2}\left(p_{1 R}^{2}+p_{1 I}^{2}\right), \\
D & =2 p_{1 R}^{2}\left(-1_{1}^{2}+l_{2}^{2}+\theta_{0}\right)-8 l_{1} l_{2} p_{1 R} p_{1 I}+2 p_{1 I}^{2}\left(l_{1}^{2}-l_{2}^{2}+\theta_{0}\right) .
\end{aligned}
$$

In particular, when $\gamma_{11}=0$, these semi-rational solutions become rational solutions for the DSIII equation, which possess two different dynamical behaviors:

(i) Lump solution. When $p_{1 I} \neq 0$, i.e., $p_{1}$ is complex, one can see that $q, U$, and $V$ are constants along the trajectory where

$$
\begin{aligned}
& \lambda p_{1 R} x+\frac{p_{1 R}}{p_{1 R}^{2}+p_{1 I}^{2}} y-\left(\frac{4 p_{1 R} p_{1 I}}{\left(p_{1 R}^{2}+p_{1 I}^{2}\right)^{2}}+2 p_{1 R} p_{1 I}\right) t=0, \\
& \lambda p_{1 I} x-\frac{p_{1 I}}{p_{1 R}^{2}+p_{1 I}^{2}} y-\left[\frac{2\left(p_{1 R}^{2}-p_{1 I}^{2}\right)}{\left(p_{1 R}^{2}+p_{1 I}^{2}\right)^{2}}-2\left(p_{1 R}^{2}-p_{1 I}^{2}\right)\right] t=0 .
\end{aligned}
$$

Indeed, at any given time, $(q, U, V) \rightarrow(1,0,0)$ when $(x, y) \rightarrow \infty$, thus these solutions are permanent lumps moving on the constant background.

Based on the analysis of critical points for rational solutions (37), these lumps can be classified into three patterns:

(a) Bright lump: when $0<p_{1 I}^{2} \leq \frac{1}{3} p_{1 R}^{2}$, one upward hump and two downward humps (i.e, $q$ has one global maximum point and two global minimum points), see Fig. 5(a) ;

(b) Bi-model lump: when $\frac{1}{3} p_{1 R}^{2}<p_{1 I}^{2}<3 p_{1 R}^{2}$, two upward humps and two downward humps (i.e, $q$ has two global maximum points and two global minimum points), see Fig. 5(b) ;

(c) Dark lump: when $p_{1 I}^{2} \geq 3 p_{1 R}^{2}$, two upward humps and one downward hump (i.e, $q$ has one global maximum point and two global minimum points), see Fig. 5 (c).

Three patterns of the first-order lump are plotted in Fig. 5. This classification is also suitable for higher order lump solutions.

(ii) Rogue wave solution. When $p_{1 I}=0, p_{1 R} \neq \pm 1$, i.e., $p_{1}$ is an arbitrary real parameter except \pm 1 , this solution is a line rogue wave. As can be seen in Fig. 6, this solution describes a line wave, which possesses a varying height. When $t \rightarrow \pm \infty$, this line wave approaches towards the constant background (see $t= \pm 10$ panel ). In the intermediate time, it attains a higher amplitude (see $t=0$ panel ). Specially, the maximum amplitude is three times the 


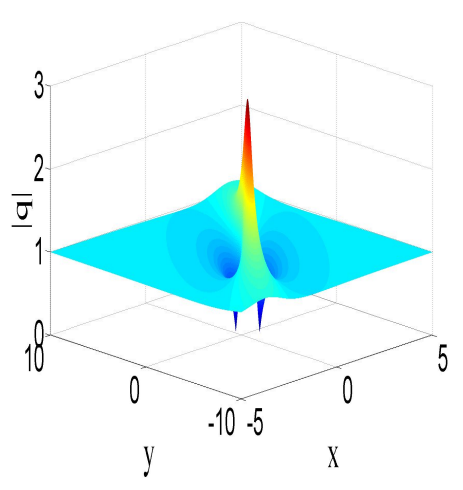

(a)

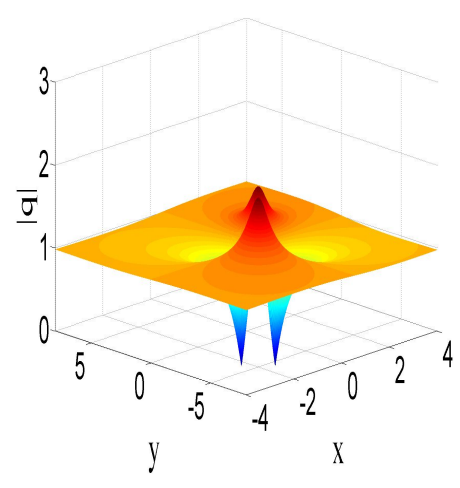

(b)

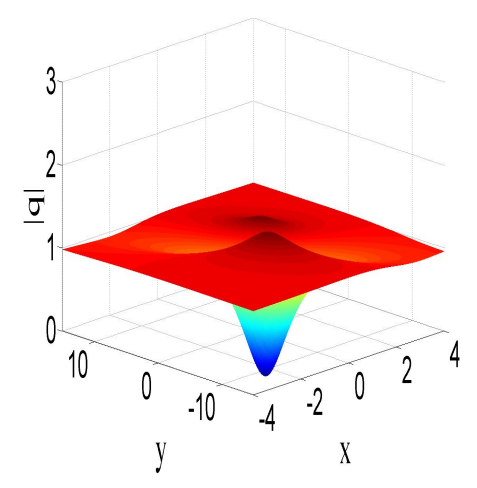

(c)

FiguRE 5. Three different patterns of fundamental lumps of the DSIII equation given by equation (37) with parameters $\gamma_{11}=0, \lambda=1, c_{11}=0$ : (a) A bright lump with parameters $p_{1}=2+i$; (b) A bi-model lump with parameters $p_{1}=1+i$;(c) A dark lump with parameters $p_{1}=1+2 i$.

constant background amplitude, which is same with the Peregrine rogue wave solutions of $(1+1)$-dimensional systems.

In contrast to the orientation direction of fundamental line rogue waves of the DSI equation, the orientation direction of this line rogue wave is almost arbitrary, while the former one has a limited range of orientations. It is noted that solutions defined in equation (37) are independent of $t$ when $p_{1}= \pm 1$, and they maintain a perfect line profile during their propagation in the $(x, y)$-plane without any decay. In this case, this solution is a rational soliton (i.e., W-shaped soliton) of the DSIII equation.

Next, we consider the case of $\gamma_{11}=1$ in equation (37). In this case, this solution also has two typical behaviours.

(a) A hybrid of rogue waves and dark solitons. When $p_{1 I}=0, p_{1 R} \neq \pm 1$, i.e., $p_{1}$ is an arbitrary real parameter except \pm 1 , this solution describes the interaction of a line rogue wave and a dark soliton. As shown in Fig. 7, when $t \rightarrow \pm \infty$, this solution describes a dark soliton, and the rogue wave approaches to the constant background (see $t= \pm 10$ panel ). In the intermediate time, a line rogue wave arises from the constant background ( see the panel at $t=0$ ), and then disappears into the constant background at larger time. It is worthful to note that the fundamental rogue wave does not exist on the whole background, but only one side of the dark solion (see the panel at $t=0$ ). May be the interaction between the dark soliton and the rogue wave destroy the wave structure of the rogue wave, and energy transfer between the dark soliton and the line rogue is also observed. Specifically, when $p_{1}= \pm 1$, the corresponding solution defined in (37) describes the interaction between a W-shaped soliton and a dark soliton, which features similar to the wave patterns in Fig.1 (c).

(b) A hybrid of lumps and dark solitons. When $p_{1 I} \neq 0$, i.e., $p_{1}$ is complex, the corresponding solution is a mixture of lumps and dark solitons. As can be seen in Fig. 8, this solution is a dark soliton when $t \rightarrow-\infty$ (see the $t=-10$ panel). In the intermediate time, a lump forms on the dark soliton, and travels with higher speed than the line soliton. Hence, at larger time, the original soliton disintegrates into a lump and a soliton ( see the panel at $t=10$ ). Obviously, these explicit solutions possess the unique dynamics simulated by numerical studies in Ref. [57,58. Besides, at this juncture, this kind of lump-line soliton solutions is quite different 


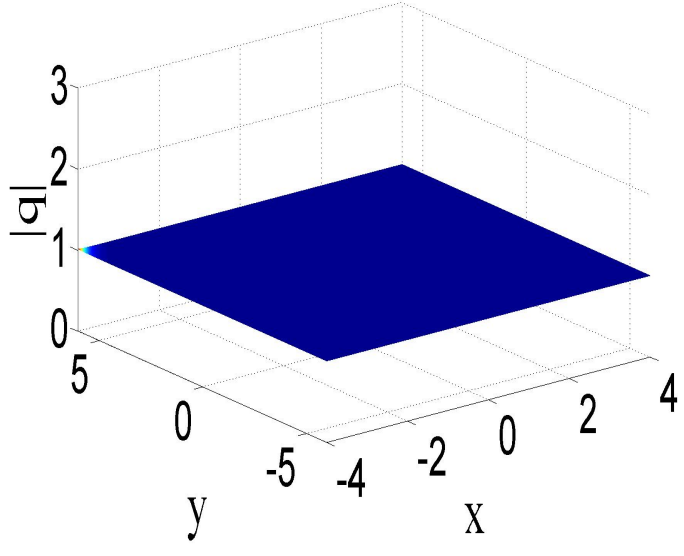

(a) $t=-10$

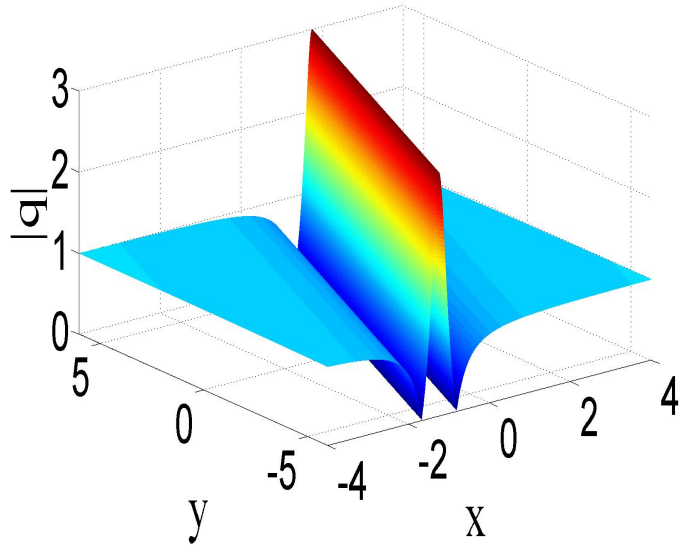

(c) $t=0$

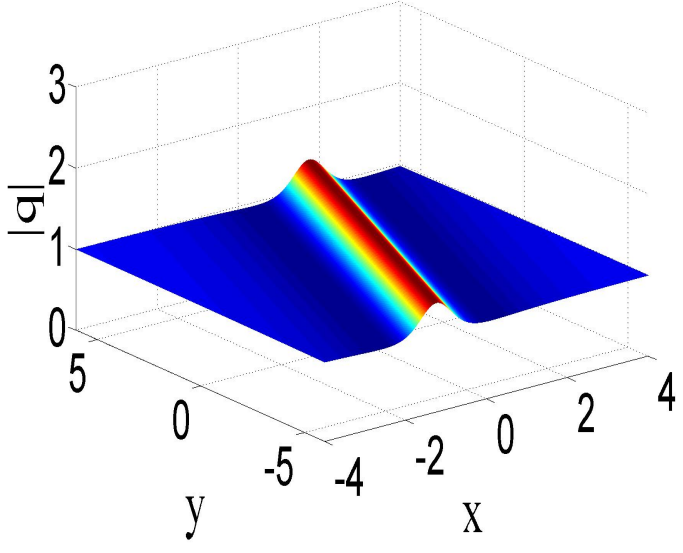

(b) $t=-1$

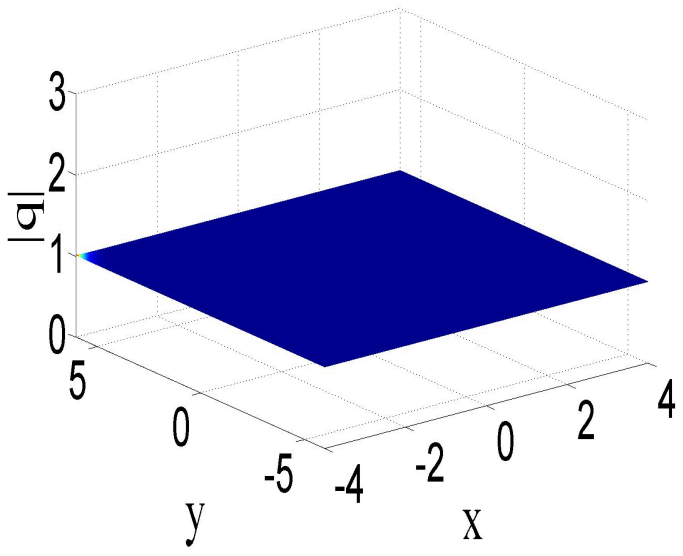

(d) $t=10$

FiguRE 6. The time evolution of fundamental line rogue waves of the DSIII equation given by equation (37) with parameters $\gamma_{11}=0, \lambda=1, p_{1}=2, c_{11}=0$.

from the lump-line soliton solutions of the DSII equation derived by Fokas et al. [75], as the latter describes lump and line soliton for all times.

Two subclasses of non-fundamental semi-rational solutions can be derived from equation (31) by taking $N>1, n_{i}=1$ or $N=1, n_{i}>1$ respectively. Below, we focus on these two subclasses of non-fundamental semi-rational solutions.

\subsection{Dynamics of Multi-semi-rational solutions.}

The first subclass of non-fundamental semi-rational solutions is obtained by taking $N>$ $1, n_{i}=1$ in equation (31), which are multi-semi-rational solutions. For different parameter choices of $\gamma_{i j}, p_{i}$, these multi-semi-rational solutions can be classified into three different patterns:

(1) Multi-rogue waves. When $p_{i}$ are real except \pm 1 and $\gamma_{i j}=0$, this semi-rational solution becomes non-fundamental rational solution for the DSIII equation, which is multi-rogue waves of the DSIII equation. 


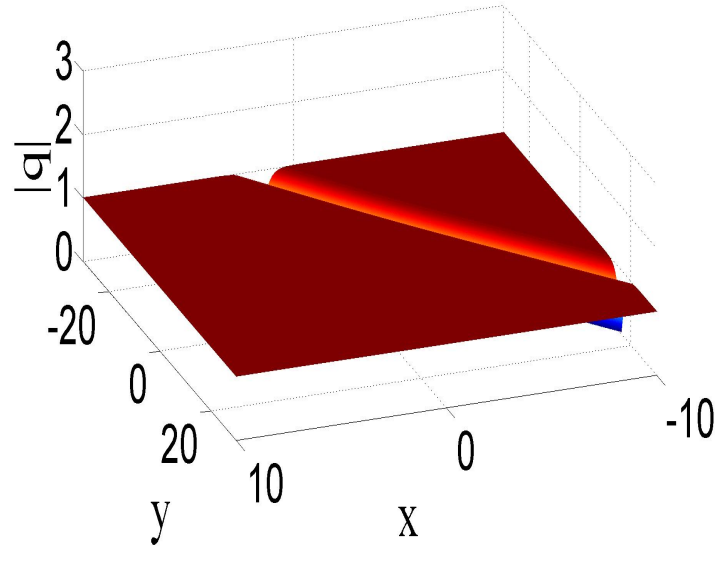

(a) $t=-10$

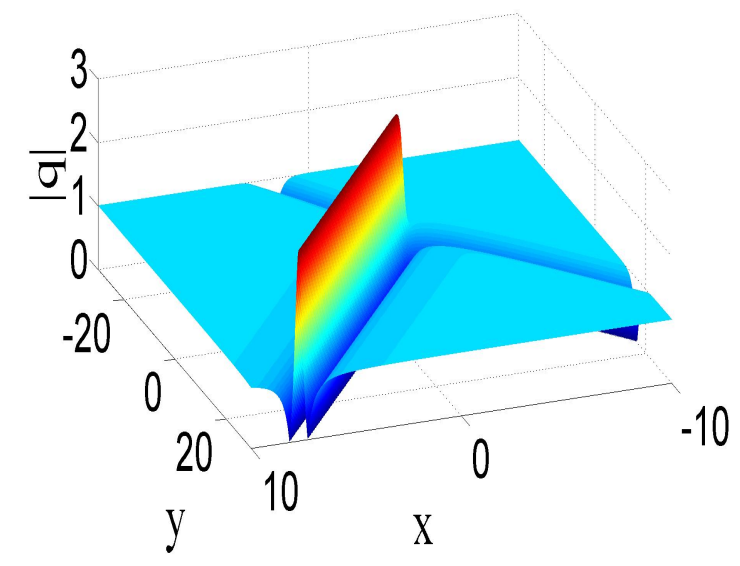

(c) $t=0$

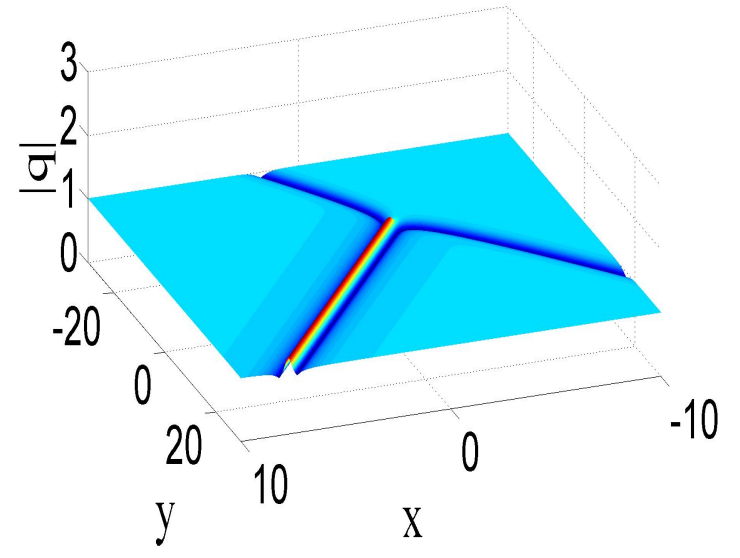

(b) $t=-1$

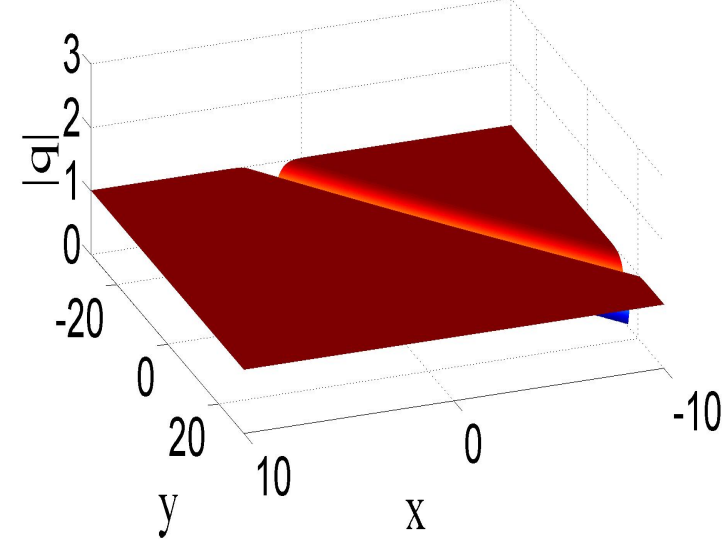

(d) $t=10$

Figure 7. The time evolution of semi-rational solutions (37) consisting of a fundamental line rogue wave and a dark soliton in the DSIII equation with parameters $\gamma_{11}=1, \lambda=1, p_{1}=2, c_{11}=10^{-4}, \xi_{10}=0$.

(2) A hybrid of multi-rogue-waves and dark solitons. When $\gamma_{i i}=1, \gamma_{i j}=0(i \neq j)$, the corresponding semi-rational solution is a combination of rogue waves and dark solitons.

(3) A hybrid of multi-rogue-waves, breathers and dark solitons. When $\gamma_{i j}=1$, the corresponding solution is a mixture of multi-rogue waves, breathers and solitons.

To demonstrate this subclass of semi-rational solutions in detail, we first consider the case of $N=2$. In this case, solutions can be derived from Theorem 2 as

$$
q=\frac{\left|\begin{array}{ll}
M_{11}^{(1)} & M_{12}^{(1)} \\
M_{21}^{(1)} & M_{22}^{(1)}
\end{array}\right|}{\left|\begin{array}{ll}
M_{11}^{(0)} & M_{12}^{(0)} \\
M_{21}^{(0)} & M_{22}^{(0)}
\end{array}\right|}, V=-\lambda \log \left(\left|\begin{array}{cc}
M_{11}^{(0)} & M_{12}^{(0)} \\
M_{21}^{(0)} & M_{22}^{(0)}
\end{array}\right|\right)_{y y}, U=-\lambda \log \left(\left|\begin{array}{cc}
M_{11}^{(0)} & M_{12}^{(0)} \\
M_{21}^{(0)} & M_{22}^{(0)}
\end{array}\right|\right)_{x x},
$$




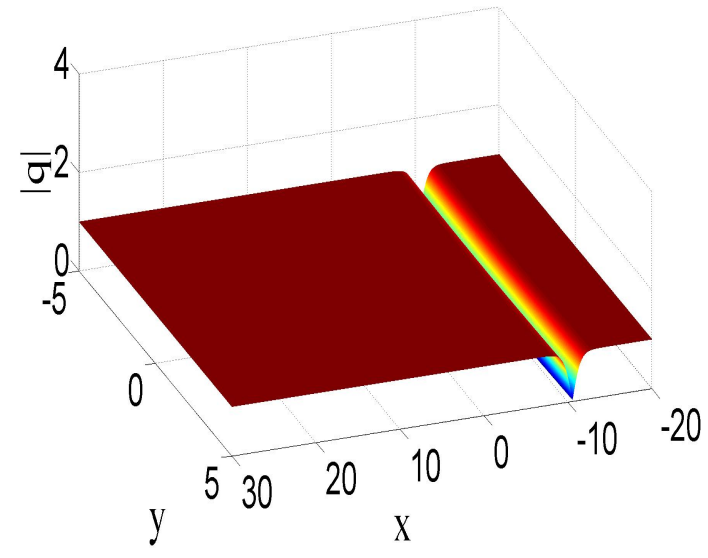

(a) $t=-10$

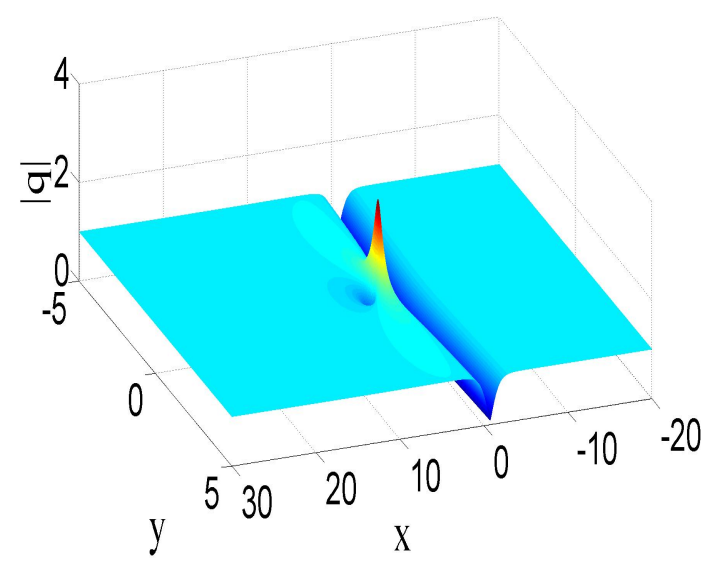

(c) $t=1$

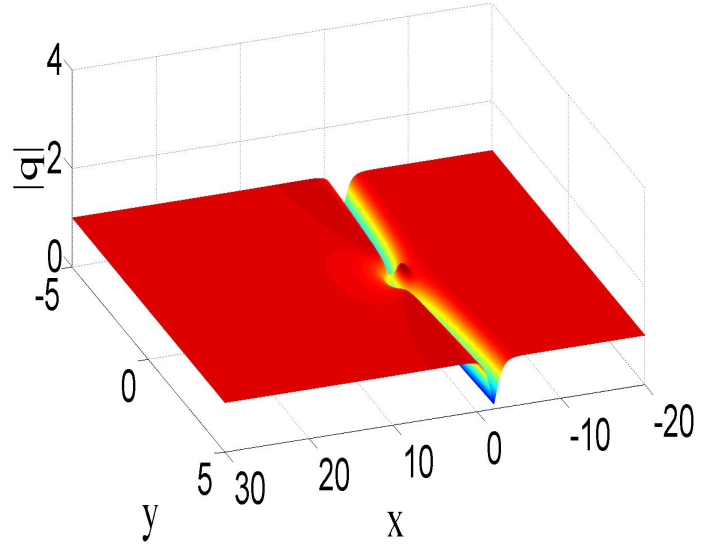

(b) $t=0$

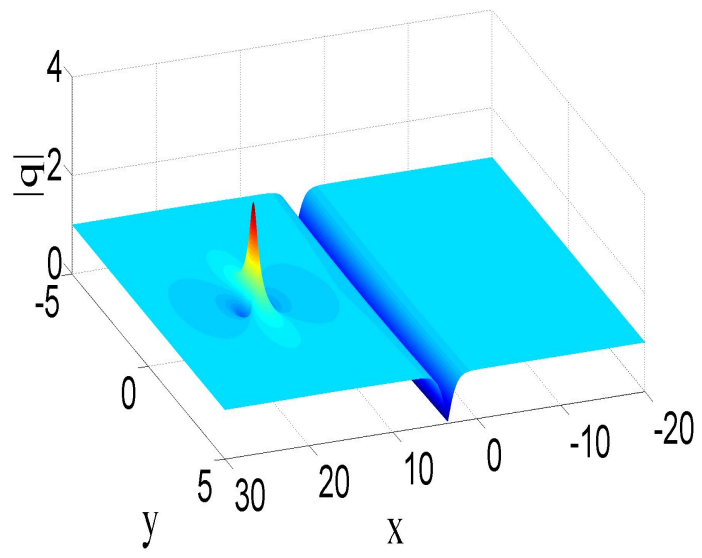

(d) $t=10$

Figure 8. The time evolution of semi-rational solutions (37) consisting of a lump and a dark soliton of the DSIII equation with parameters $\gamma_{11}=1, \lambda=$ $1, p_{1}=\frac{2}{3}+\frac{1}{3} i, c_{11}=10^{-4}, \xi_{10}=0$.

where

$$
M_{i j}^{(n)}=\frac{e^{\xi_{i}+\xi_{j}^{*}}}{p_{i}+p_{j}^{*}}\left[\left(\xi_{i}^{\prime}-\frac{p_{i}}{p_{i}+p_{j}^{*}}+c_{i 1}+n\right)\left(\xi_{j}^{*}-\frac{p_{j}^{*}}{p_{i}+p_{j}^{*}}+c_{i 1}^{*}-n\right)+\frac{p_{j}^{*}}{\left(p_{i}+p_{j}^{*}\right)^{2}}\right]+\gamma_{i j} c_{i 1} c_{j 1}^{*},
$$

and $\xi_{i}, \xi_{i}^{\prime}$ are given in Theorem 2. These solutions possess different dynamics determined by parameters $\gamma_{i j}$ and $p_{i}$.

(i) When one takes $\gamma_{i j}=0$ and real parameters $p_{i}\left(p_{i} \neq 1\right)$, this solution is actually the two-rogue wave solution of the DSIII equation. This solution with parameters

$$
p_{1}=2, p_{2}=\frac{3}{2}, c_{11}=0, c_{21}=0
$$

is shown in Fig.9. As can be seen from figure, two crossed line rogue waves arise from the constant background, and the interaction region attains much higher amplitudes, the wave patterns are no longer straight lines until these line waves disappear into the constant background. It is 
noted that these two line rogue wave would not be separated for all times. As non-fundamental rogue waves of the DSIII equation in Ref. [71] are parallel line rogue waves, thus this two-rogue wave of the DSIII equation in our paper is quite different.

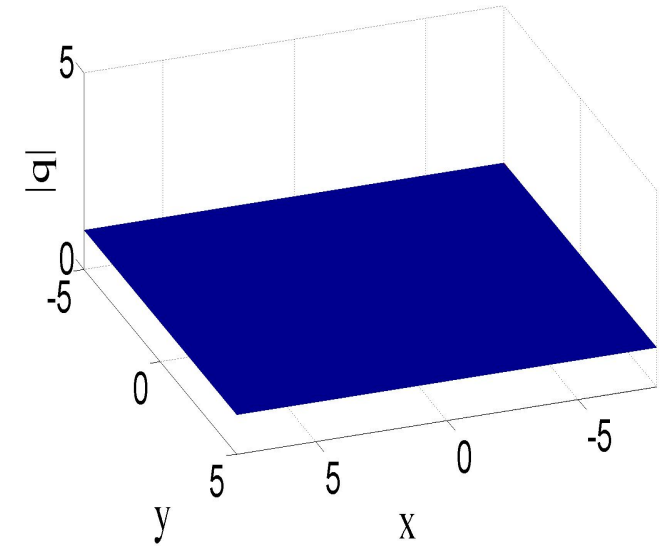

(a) $t=-10$

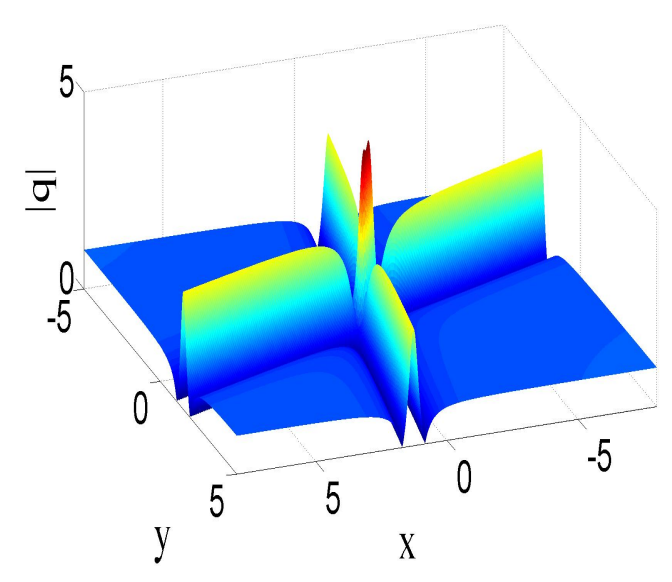

(c) $t=0$

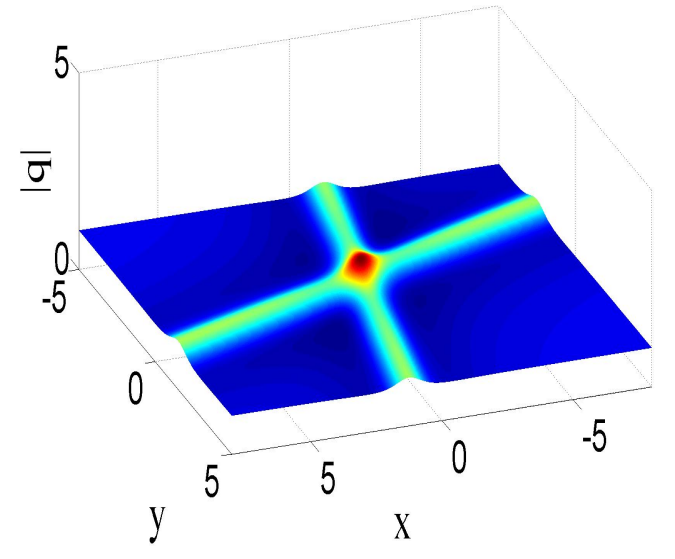

(b) $t=-1$

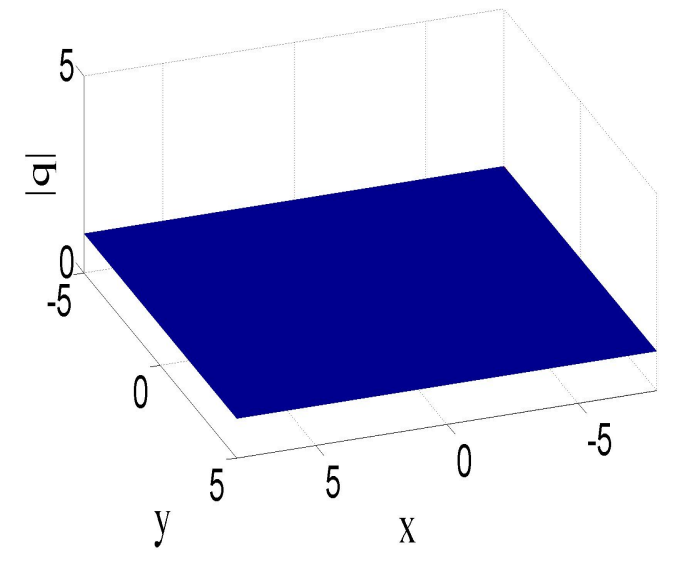

(d) $t=10$

FiguRE 9. The time evolution of multi-rogue waves of the DSIII equation defined in equation (39) with parameters (41) and $\gamma_{11}=0, \gamma_{12}=0, \gamma_{21}=0, \gamma_{22}=$ $0, c_{11}=0, c_{21}=0$.

Specially, when $p_{1}=1, p_{2} \neq 1$, this solution becomes a hybrid of one rogue wave and one $W$-shaped soliton, which possess different dynamics comparing to the two-rogue wave shown in Fig.9, see Fig,10. It is seen that, when a line rogue wave arise from the constant background, the $W$-shaped soliton is not a continuous line any more, and starts to disconnect into two part of lines gradually until they separate completely (see the $t=0$ panel). In the intermediate time, this rational solution features the same wave pattern as multi-rogue waves in DSI equation (the $t=0$ panel of Fig.2 in Ref. [67]). At $t \rightarrow+\infty$, this solution turns out to be a $W$-shaped line soliton again.

For larger $N$ and $\gamma_{i j}=0$ with real $p_{i}$, these higher-order rogue waves behave qualitatively similar as the second-order rogue waves shown in Fig. 9, but more rogue waves interact with 


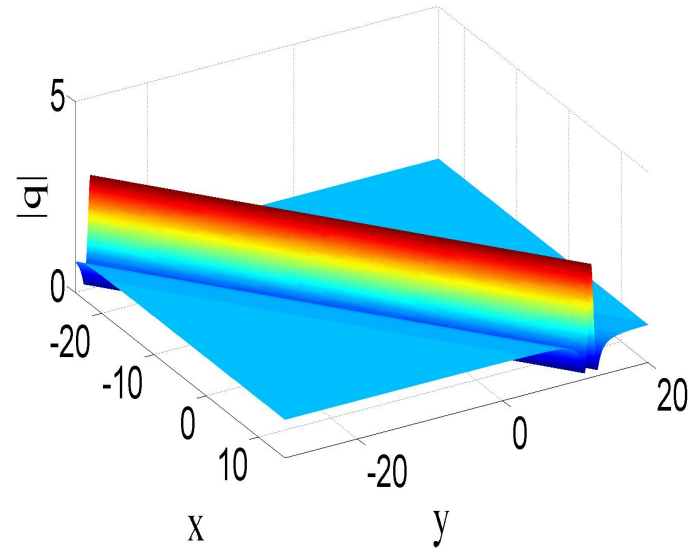

(a) $t=-10$

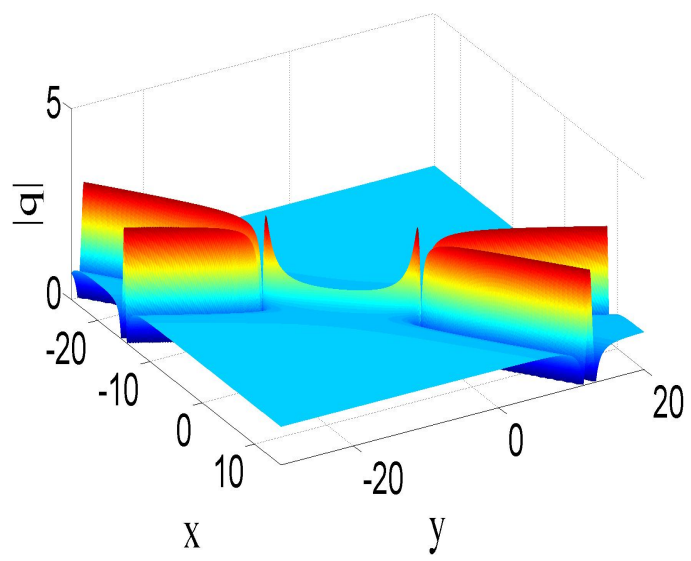

(c) $t=0$

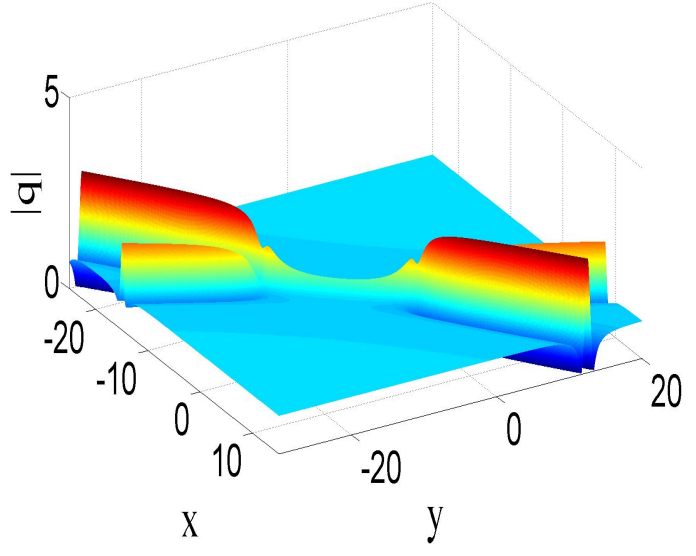

(b) $t=-1$

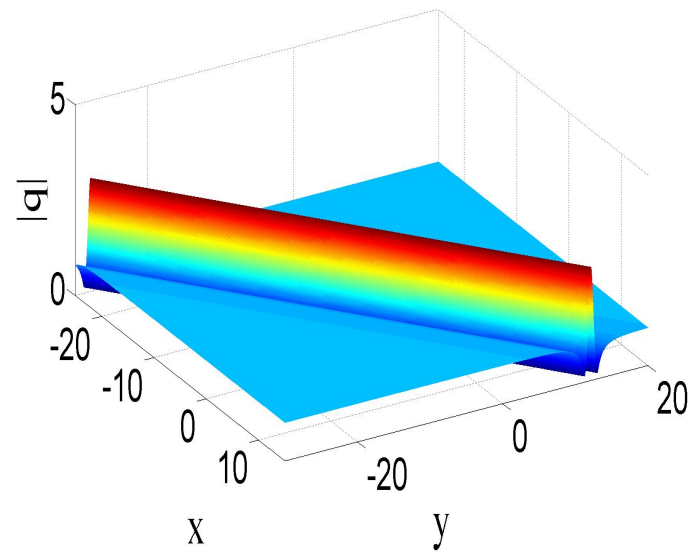

(d) $t=10$

FiguRE 10. The time evolution of mutli-rational solutions consisting of a fundamental rogue wave and a $W$-shaped soliton of the DSIII equation given by equation (39) with parameters $p_{1}=1, p_{2}=2, \gamma_{11}=0, \gamma_{12}=0, \gamma_{21}=0, \gamma_{22}=$ $0, c_{11}=0, c_{21}=0$.

each other, and the wave patterns become more complicated. Besides, they always hold the characteristics "appear from nowhere and disappear without a trace" [2]. The Fig. 11] shows a third-order rogue wave with parameters

$$
N=3, \lambda=1, p_{1}=\frac{1}{2}, p_{2}=\frac{2}{3}, p_{3}=\frac{3}{2}, \gamma_{i j}=0, c_{i 1}=0(1 \leq i, j \leq 3) .
$$

Note that the maximum value of the solution $|q|$ can exceed 5 (i.e., five times the constant background) at some times, . Thus this interaction between these tree line rogue waves can generate higher peaks.

(ii) When one takes $\gamma_{i i}=1, \gamma_{i j}=0(i \neq j)$ and real parameters $p_{i}\left(p_{i} \neq 1\right)$ in equation (39), this solution demonstrates the second-order rogue waves on a two-dark-soliton background. However, when one takes $\gamma_{i i}=1, \gamma_{i j}=0(i \neq j)$ and complex parameters $p_{i}$ in equation (39), this solution describes two-dark soliton when $t \rightarrow-\infty$, see Fig. 12. In the intermediate time, 


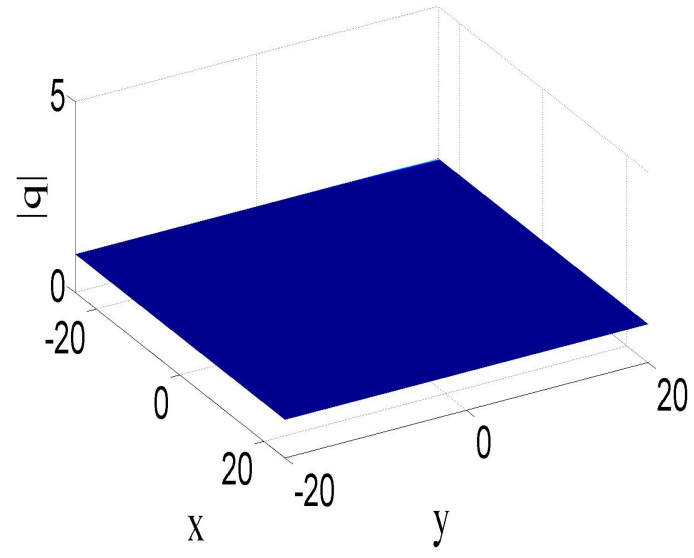

(a) $t=-10$

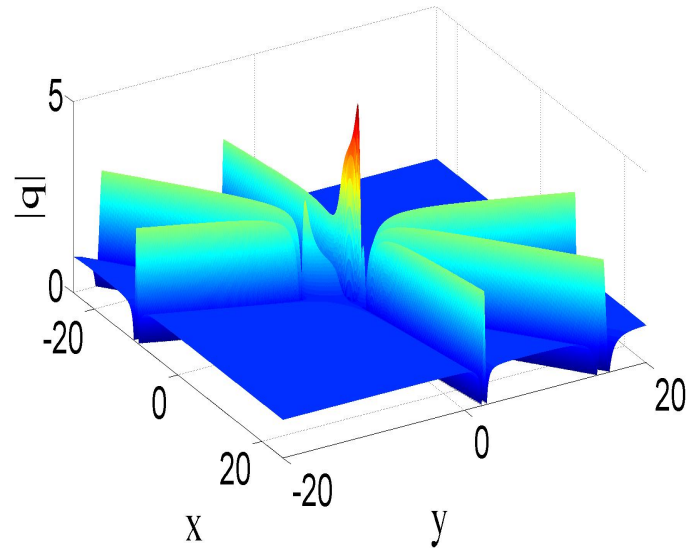

(c) $t=0$

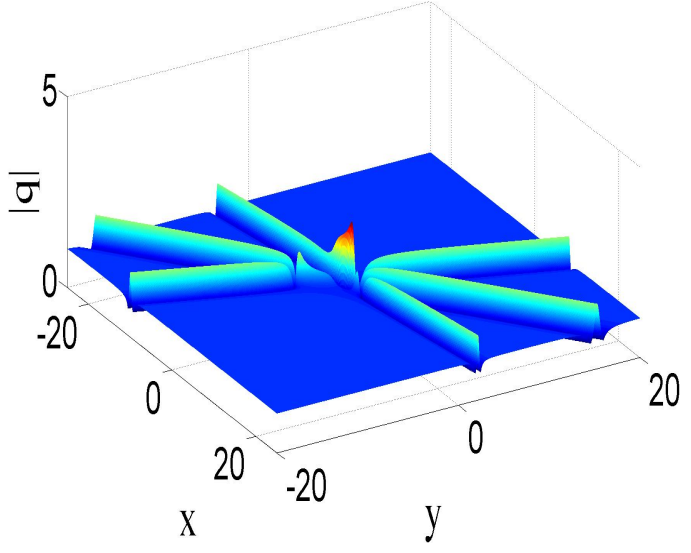

(b) $t=-1$

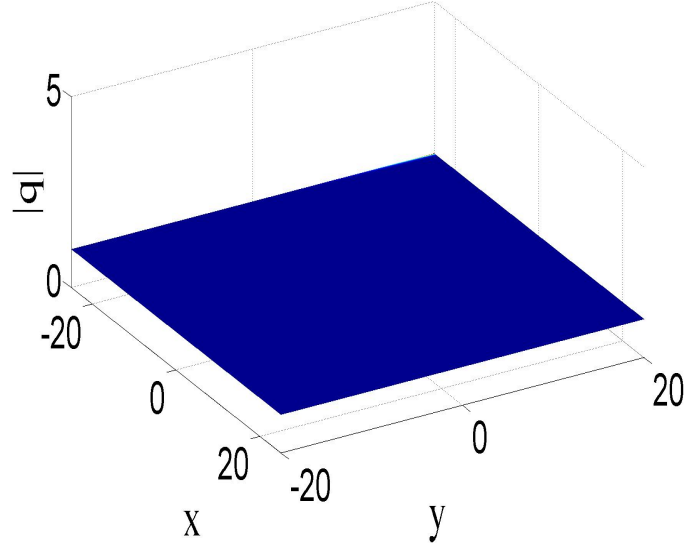

(d) $t=10$

FiguRE 11. The time evolution of a third-order rogue wave of the DSIII equation with parameters given by equation (42).

two lumps form on the two-dark soliton gradually (see the panels at $t=0,1$ ). When $t \rightarrow+\infty$, the original two-dark soliton develops into two-dark soliton and two lumps (see the panel at $t=10)$.

(iii) When one takes $\gamma_{i j}=1(1 \leq i, j \leq 2)$ and complex parameters $p_{i}$ satisfies $p_{1}=p_{2}^{*}$, this solution is a hybrid of two lumps, one dark soliton and a line breather. This solution with parameters

$$
N=2, \lambda=1, p_{1}=1+\frac{1}{2} i, p_{2}=1-\frac{1}{2} i, \gamma_{i j}=1, c_{i 1}=0(1 \leq i, j \leq 2),
$$

is shown in Fig.13. As can be seen, when $t \rightarrow \pm \infty$, this solution is only composed of two lumps and a dark soliton ( see the panels at $t= \pm 10$ ). Differently, these two lumps exist on the background for all times, which form on the dark soliton as shown in Fig, 12 . In the intermediate time, the line breather arises from the constant background, and retreat back to it at larger time (see the panels at $t=-1,0$ ). During this short period, this solution is made 


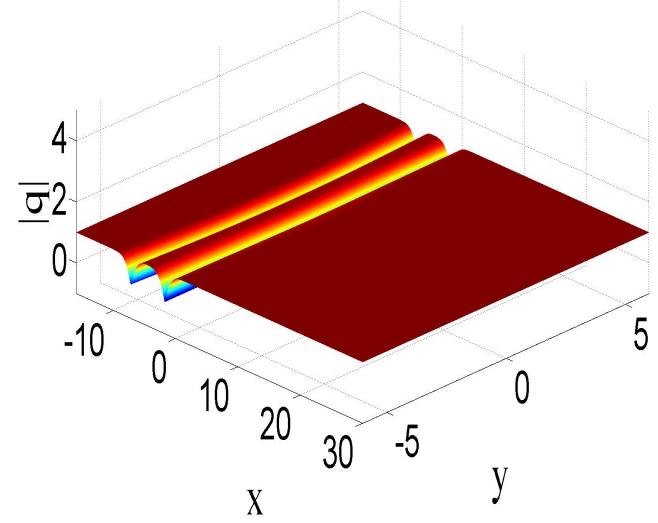

(a) $t=-10$

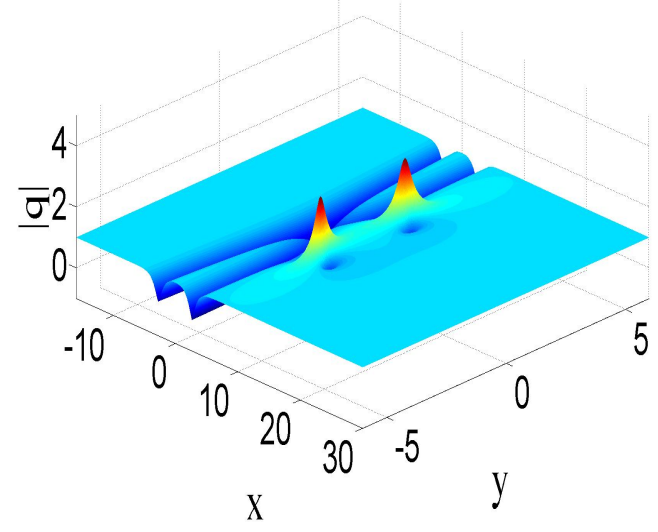

(c) $t=1$

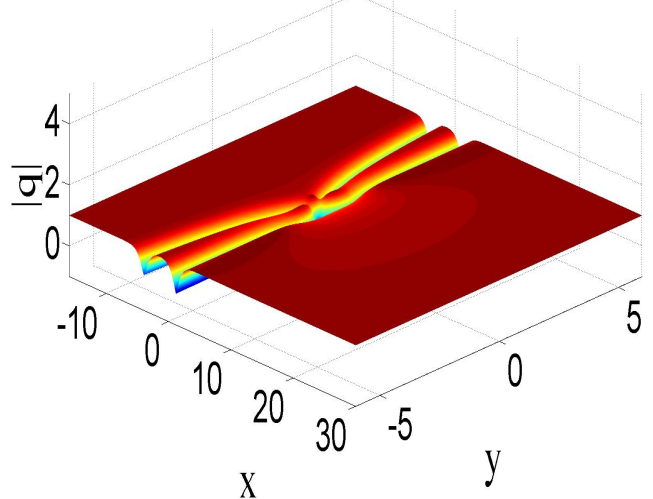

(b) $t=0$

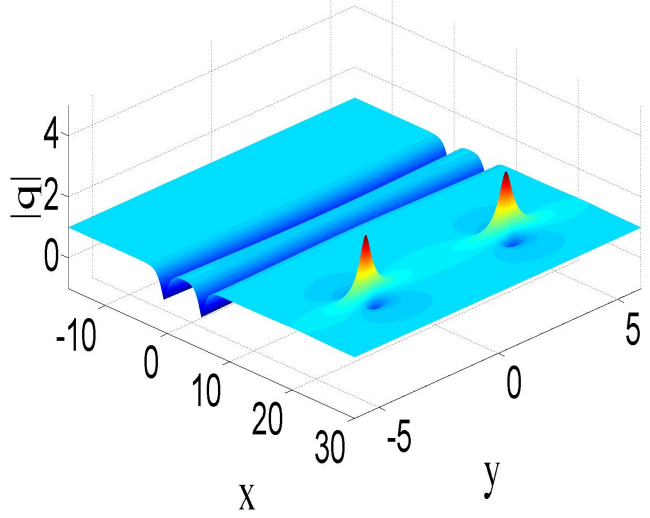

(d) $t=10$

Figure 12. The time evolution of second-order lump on a second-order dark soliton background of the DSIII equation with parameters $\gamma_{11}=\gamma_{22}=1, \gamma_{12}=$ $\gamma_{21}=0, \lambda=1, p_{1}=2+i, p_{2}=2+\frac{9}{10} i, c_{11}=10^{-4}, c_{21}=10^{-4}, \xi_{10}=\xi_{20}=0$.

up of two lumps, a dark soliton and one line breather. To the best of our knowledge, this kind of mixed solution has not been reported before.

\subsection{Higher-order semi-rational solutions.}

Another subclass of non-fundamental semi-rational solutions is the higher-order semi-rational solutions, which can be derived by taking $N=1, n_{i}>1$ in equation (30). This subclass of semi-rational solutions can be classified into different patterns:

(a) Higher order rogue waves. When one takes $\gamma_{11}=0$ and real parameter $p_{1}$ except \pm 1 in equation (30), the corresponding semi-rational solutions become rational solutions, which is the higher order rogue waves in the DSIII equation.

(b) Higher order rogue waves on a dark soliton background. When one takes $\gamma_{11}=1$ and real parameter $p_{1}$ except \pm 1 , the semi-rational solutions are mixed solutions consisting of higher 


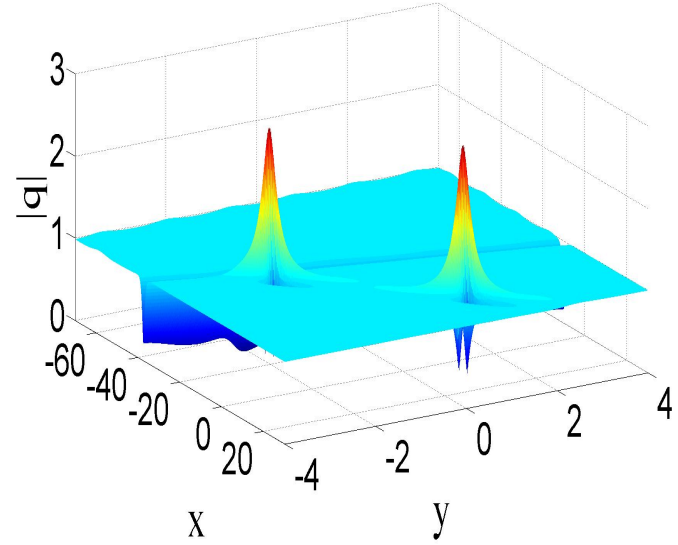

(a) $t=-10$

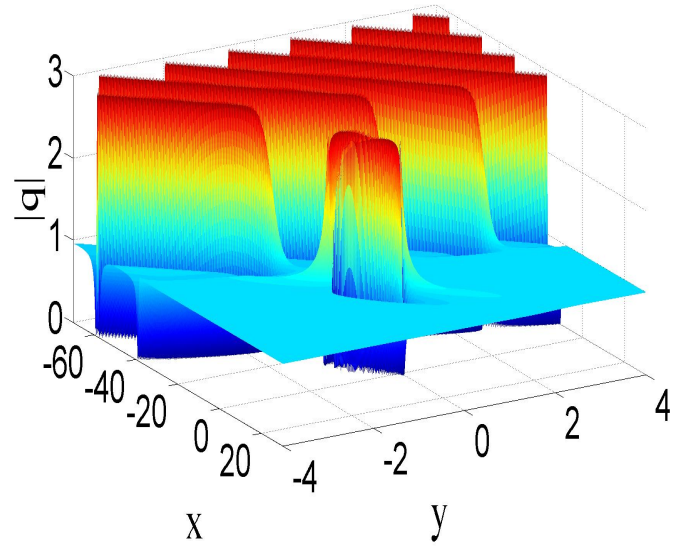

(c) $t=0$

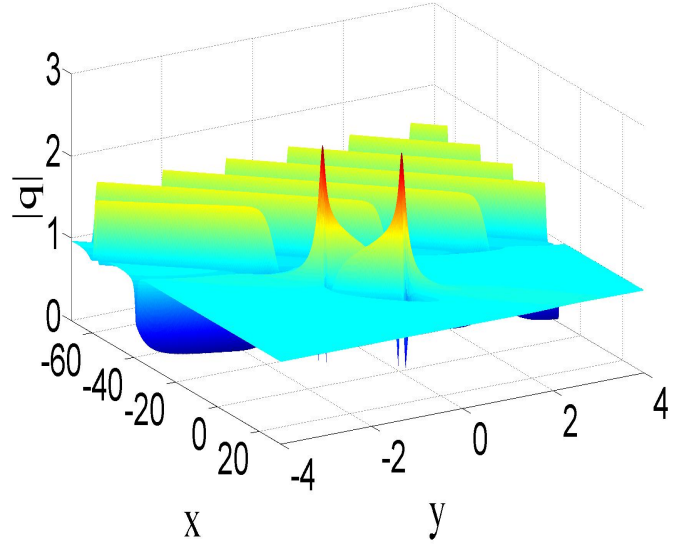

(b) $t=-1$

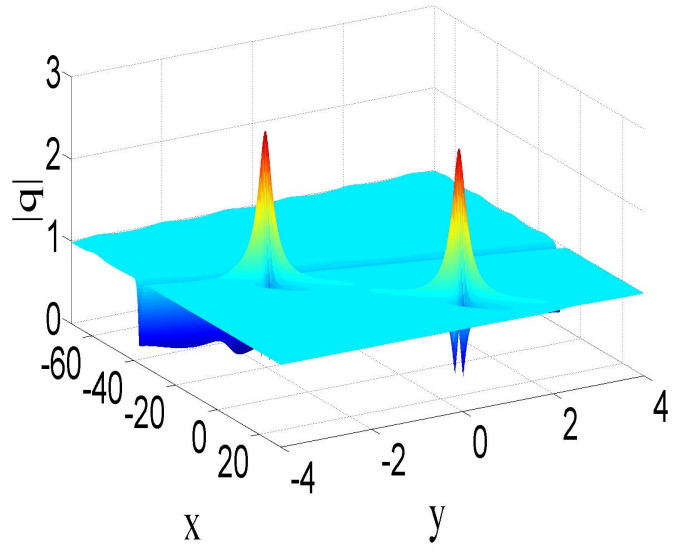

(d) $t=10$

Figure 13. The time evolution of mutli-rational solutions 39 consisting of two lumps, a dark soliton and a line breather of the DSIII equation with parameters (43).

order rogue waves and a dark soliton. In this case, these rogue waves are also localized both in space and time in two dimensions.

(c) Higher order lumps on a dark soliton background. When one takes $\gamma_{11}=0$ and complex parameter $p_{1}$, these solutions describe $n_{i}$ lumps form on the dark soliton background gradually. When $t \rightarrow-\infty$, these semirational solutions are a dark soliton, while they are a hybrid of $n_{i}$ lumps and a dark soliton when $t \rightarrow+\infty$.

To demonstrate this subclass of non-fundamental semi-rational solutions in details, we take the case of $n_{i}=2$ as an example. In this case, the explicit forms of the second-order semirational solutions of the DSIII equation are given by the following formulae :

$$
q=\frac{g}{f}, V=-\lambda \log (f)_{y y}, U=-\lambda \log (f)_{x x}
$$


with

$$
\begin{aligned}
& f=e^{\xi_{1}+\xi_{1}^{*}}\left[\left(p_{1} \partial_{p_{1}}+\xi_{1}^{\prime}\right)^{2}+c_{12}\right]\left[\left(p_{1}^{*} \partial_{p_{1}^{*}}+\xi_{1}^{*}\right)^{2}+c_{12}^{*}\right] \frac{1}{p_{1}+p_{1}^{*}}+\gamma_{11} c_{12} c_{12}^{*}, \\
& g=e^{\xi_{1}+\xi_{1}^{*}}\left[\left(p_{1} \partial_{p_{1}}+\xi_{1}^{\prime}+1\right)^{2}+c_{12}\right]\left[\left(p_{1}^{*} \partial_{p_{1}^{*}}+\xi_{1}^{*}-1\right)^{2}+c_{12}^{*}\right] \frac{1}{p_{1}+p_{1}^{*}}+\gamma_{11} c_{12} c_{12}^{*},
\end{aligned}
$$

and $\xi_{1}, \xi_{1}^{\prime}$ are defined in Theorem 2. By different parameters $p_{1}$ and $\gamma_{11}$, these solutions can be classified into following patterns:

(1) When taking $\gamma_{11}=0$ and real parameter $p_{1}$, the corresponding solution is a secondorder rogue wave, see Fig,14. Different from the second-order rogue wave shown in Fig,9, when $t \rightarrow \pm \infty$, this rogue wave features a localized lump traveling with the constant background (see the panel $t= \pm 10$ ). In the intermediate time, the lump disappears and the rogue wave features a parabola-shaped line waves(see the panel at $t=0$ ).

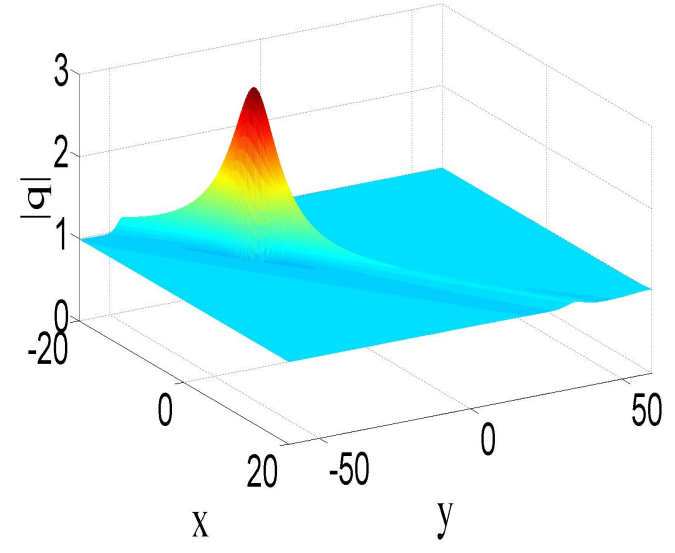

(a) $t=-10$

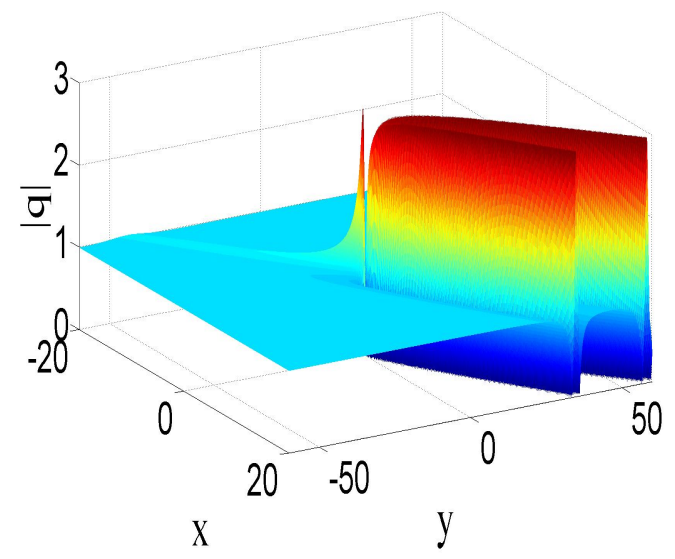

(c) $t=0$

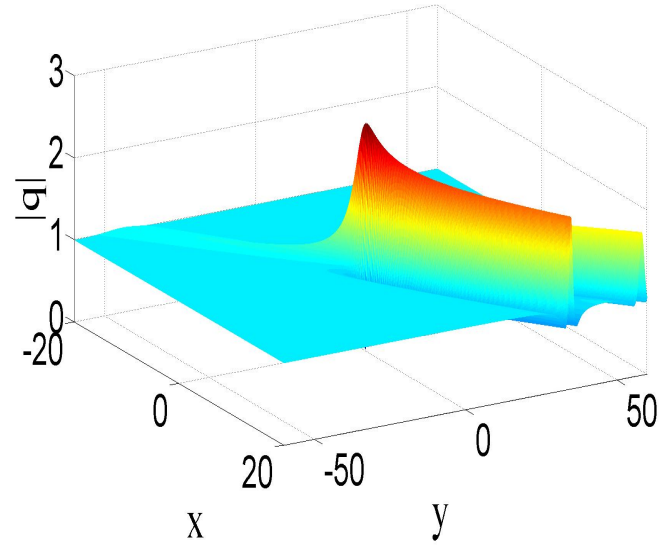

(b) $t=-1$

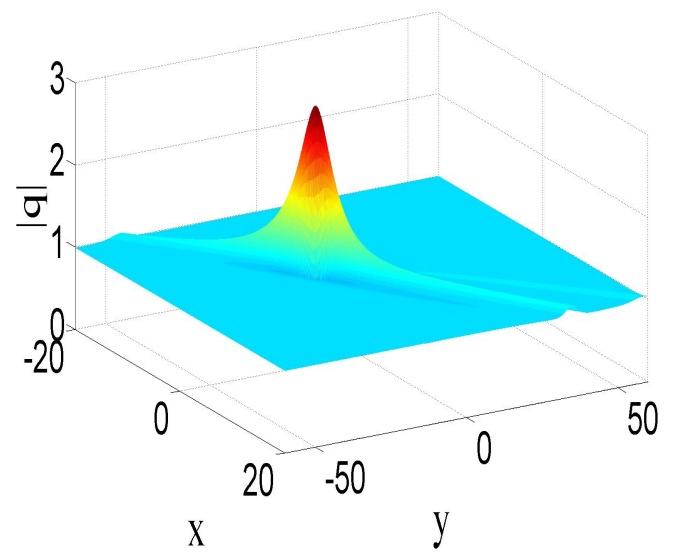

(d) $t=10$

FigurE 14. The time evolution of second-order rogue waves of the DSIII equation defined in equation (44) with parameters $\gamma_{11}=0, p_{1}=2, c_{11}=0, c_{12}=$ $0, \xi_{10}=0$. 
(2) When taking $\gamma_{11}=1$ and real parameter $p_{1}$, these semi-rational solutions consisting of a second-order rogue wave and a dark soliton possess a new phenomenon: line rogue wave localized both in space and time. As shown in Fig. 15, after the lump immerses into the line rogue wave, this second-order line rogue wave approaches maximum amplitudes. It is to be noted that this higher order rogue wave just exist in a localized space (see the panel at $t=0$ ). At larger time, it disappears into the constant background and retreat back to a lump. To the best of our knowledge, this type of line rogue waves which are localized in both space and time have never been reported before.

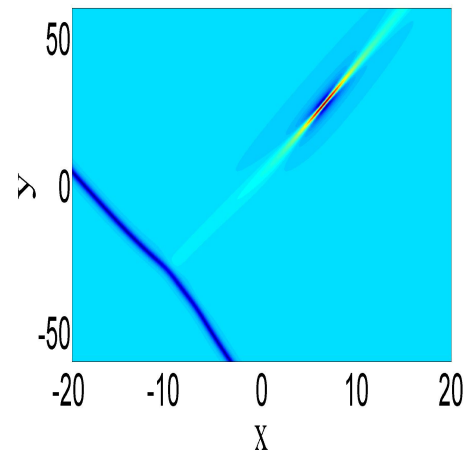

(a) $t=-10$

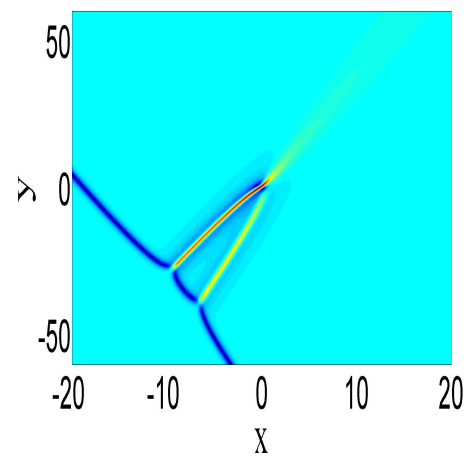

(d) $t=1$

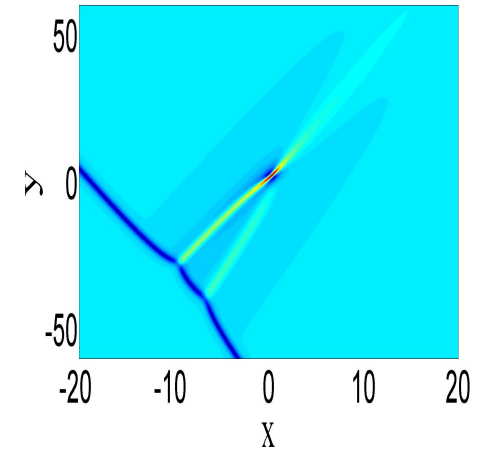

(b) $t=-1$

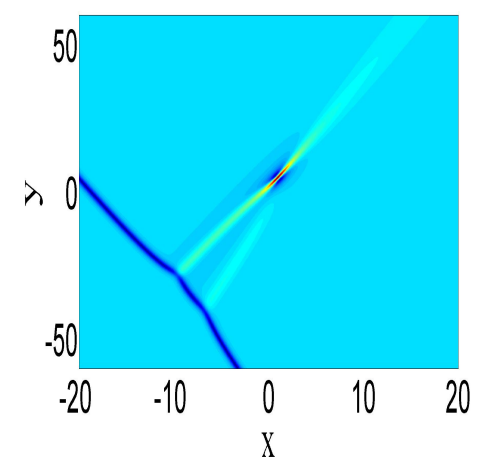

(e) $t=5$

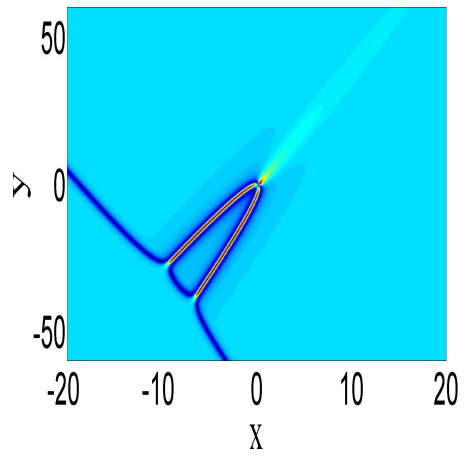

(c) $t=0$

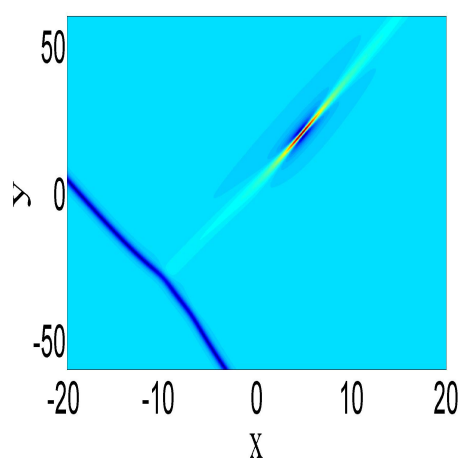

(f) $t=10$

Figure 15. The time evolution of second-order rogue waves (44) on a dark soliton background in the DSIII equation with parameters $\gamma_{11}=1, p_{1}=2, c_{11}=$ $0, c_{12}=1, \xi_{10}=0$. The plotted is $|q|$ field. The constant background value is 1 .

(3) When taking $\gamma_{11}=1$ and complex parameter $p_{1}$, in this situation, the corresponding solution describes a kind of unique dynamics, see Fig. 16. As $t \rightarrow-\infty$, this solution is a dark soliton (see the panel at $t=-10$ ). However, in the intermediate time, we observe gradual separation of two lumps separated from the dark soliton, and the original dark soliton splits into one dark soliton and two lumps as $t \rightarrow+\infty$ ( see the panel at $t=10$ ). This unique dynamical behaviour corresponds to the numerical study of blow up solutions in KP equation [57,58]. 


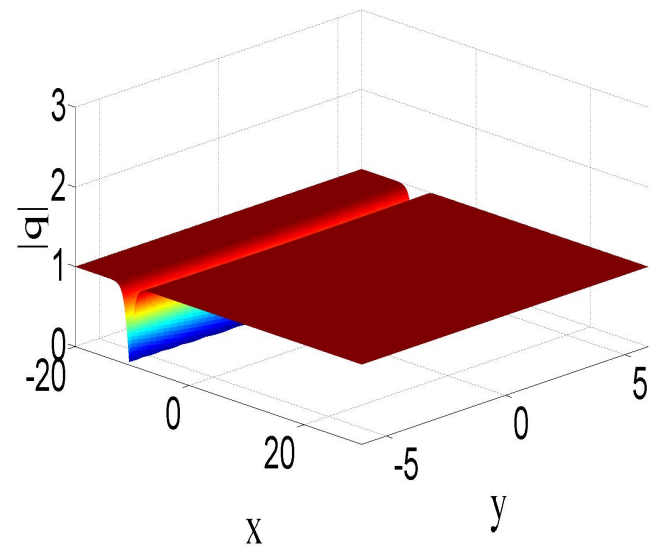

(a) $t=-10$

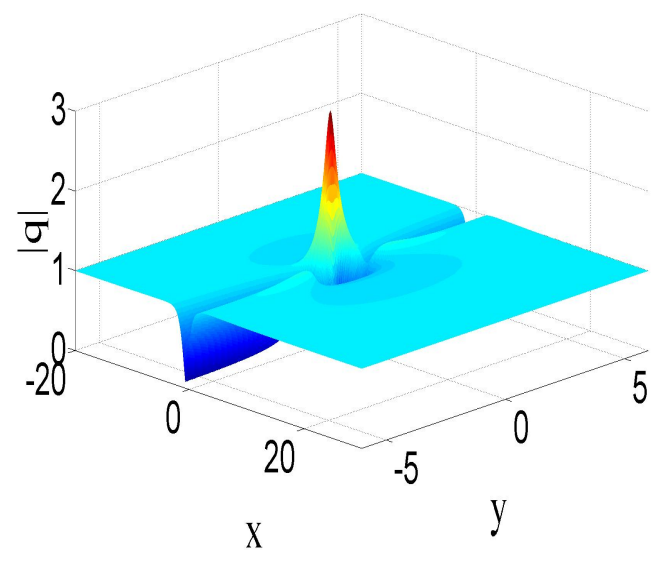

(c) $t=0$

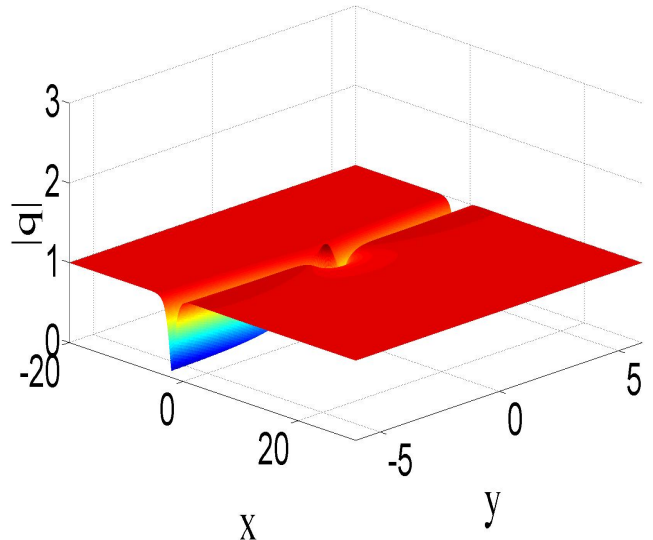

(b) $t=-1$

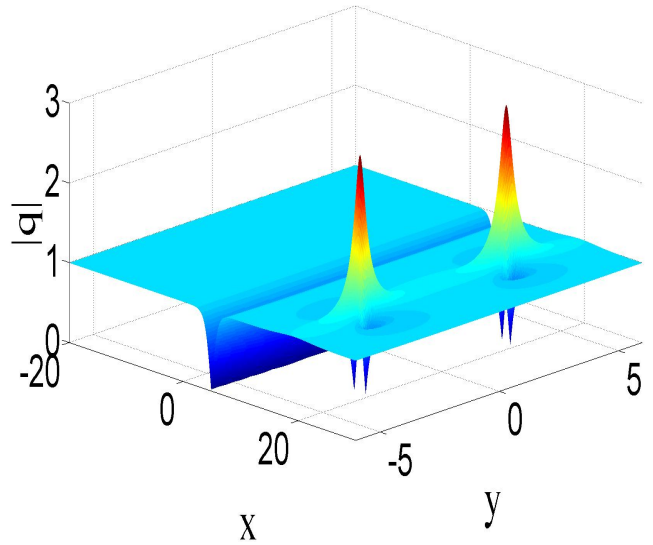

(d) $t=10$

FIgURE 16. The time evolution of high-order semi-rational solutions consisting of two lumps and a dark soliton of the DSIII equation given by equation (44) with parameters $\gamma_{11}=1, p_{1}=2+i, c_{11}=0, c_{12}=1, \xi_{10}=0$.

\section{Summary AND Discussion}

In this paper, we derived general $N$ th-order dark solitons of the DSIII equation by reducing the Gram-type solutions of the KP hierarchy. Under suitable constraint in terms of parameters, a new kind of mixed solutions consisting of usual breathers, line breathers and dark solitons are given through Gram determinants, see Fig,4. By introducing differential operators $A_{i}, B_{j}$, general semi-rational solutions containing rogue waves, breathers and dark solitons are generated. We showed that the fundamental rogue waves are line rogue waves, which arise from the constant background and retreat back to the constant background again. Except fundamental rogue waves interacting with dark solitons, these fundamental semi-rational solutions also demonstrate another unique phenomenon: a lump form on the dark soliton and separate from the dark soliton (see Fig. 8). We also showed that multi-rogue waves demonstrate the interaction of several fundamental line rogue waves, and interesting curvy wave patterns appear due to the interaction (see Fig,9 and Fig. 11). The multi-semi-rational solutions possess various 
dynamics, such as more lumps form on multi-dark solitons (see Fig,12), such as interaction of lumps, line breathers and dark solitons (see Fig. 13). Differently, the higher order rogue waves start from a localized lump and retreat back to it (see Fig,14). A new kind of line rogue waves which are localized both in space and time are illustrated, see Fig. 15. The higher order semi-rational solutions illustrating the dynamics that more lumps separated from the one soliton gradually have also been shown (see Fig. 16), these dynamics correspond to the numerical study of blow up solutions in KP equation [57, 58].

Thus, our reported results in this paper are more richer in nature when compared with the earlier reported results on the DSIII equation. Another advantage of this paper is that the expressions of obtained solutions are very simple, which are given in terms of determinants.

Acknowledgments This work is supported by the NSF of China under Grant No. 11671219, and the K.C. Wong Magna Fund in Ningbo University. We thank other members in our group at Ningbo University for their many discussions and suggestions on the paper. K.P. also acknowledges support from the National Board for Higher Mathematics (NBHM), Department of Science and Technology-Science and Engineering Research Board (DST-SERB), Indo-French Centre for the Promotion of Advanced Research IFCPAR (5104-2) and Council of Scientific and Industrial Research (CSIR), Government of India.

\section{REFERENCES}

[1] K. Dysthe, H.E. Krogstad, P. Müller, Annu. Rev. Fluid Mech. 40, 287(2008).

[2] N. Akhmediev, A. Ankiewicz and M. Taki, Phys. Lett. A 373, 675(2009).

[3] C. Garrett and J. Gemmrich, Phys. Today 62, 62(2009).

[4] E. Pelinovsky and C. Kharif, Extreme Ocean Waves (Springer, Berlin, Heidelberg, 2008).

[5] A. R. Osborne, Nonlinear Ocean Waves and The Inverse Scattering Transform (Academic Press, New York, 2010).

[6] Y. V. Bludov, V. V. Konotop, and N. Akhmediev, Phys. Rev. A 80, 033610(2009)

[7] Y. V. Bludov, V. V. Konotop, and N. Akhmediev, Eur. Phys. J. Spec. Top. 185, 169(2010).

[8] A. Montina, U. Bortolozzo, S. Residori, and F. T. Arecchi, Phys. Rev. Lett. 103, 173901(2009).

[9] D. R. Solli, C. Ropers, P. Koonath, and B. Jalali, Nature(London) 450, 1054 (2007).

[10] R. Höhmann, U. Kuhl, H. J. Stöckmann, L. Kaplan, and E. J. Heller, Phys. Rev. Lett. 104, 093901(2010).

[11] A. N. Ganshin, V. B. Efimov, G. V. Kolmakov, L.P. Mezhov-Deglin, and P. V. E. McClintock, Phys. Rev. Lett. 101, 065303(2008).

[12] W. M. Moslem, Phys. Plasmas 18, 032301 (2011).

[13] H. Bailung, S. K Sharma, and Y. Nakamura, Phys. Rev. Lett. 107, 255005(2011).

[14] T. P. Horikis and M. J. Ablowitz, Phys. Rev. E, 95, 042211(2017).

[15] F. Fedele, Journal of Fluid Mechanics 782, 25(2015).

[16] M. A. Tayfun, and F. Fedele, Ocean Engineering 34, 1631(2007).

[17] C. Lecaplain, P. Grelu, J. M.Soto-Crespo, and N. Akhmediev, Phys. Rev. Lett. 108 233901(2012).

[18] A. N. Pisarchik, R. Jaimes-Reátegui, R. Sevilla-Escoboza, G. Huerta-Cuellar and M. Taki, Phys. Rev. Lett. 107, 274101(2011).

[19] D. H. Peregrine, J. Aust. Math. Soc. B 25, 16(1983).

[20] P. Dubard and V. B. Matveev, Nat. Hazards Earth. Syst. Sci. 11, 667(2011).

[21] N. Akhmediev, A. Ankiewicz, and J. M. Soto-Crespo, Phys. Rev. E. 80, 026601(2009).

[22] P. Dubard, P. Gaillard, C. Klein, and V. B. Matveev, Eur. Phys. J. Spec. Top. 185, 247(2010).

[23] A. Ankiewicz, D. J. Kedziora, and N. Akhmediev, Phys. Lett. A375, 2782(2011).

[24] B. Guo, L. Ling, and Q. P. Liu, Phys. Rev. E 85, 026607(2012).

[25] D. J. Kedziora, A. Ankiewicz, and N. Akhmediev, Phys. Rev. E 84, 056611(2011).

[26] Y. Ohta and J. K. Yang, Proc. R. Soc. A 468, 1716(2012).

[27] J. S. He, H. R. Zhang, L. H. Wang, K. Porsezian, and A. S. Fokas, Phys. Rev. E 87, 052914(2013).

[28] F. Baronio, M. Conforti, A. Degasperis and S. Lombardo, Phys. Rev. Lett. 111, 114101(2013).

[29] X. Wang, J. I. Cao and Y. Chen, Phys. Scr. 90 105201(2015). 
[30] S. H. Chen, J. M. S. Crespo, and P. Grelu, Opt. Express. 23 349(2015).

[31] G. Mu, Z. Y. Qin, Nonlinear Anal. Real World Appl. 31, 179(2016).

[32] S. H. Chen, Phys. Rev. E 5, 023202(2013).

[33] U. Bandelow and N. Akhmediev, Phys. Lett. A 376, 1558(2012).

[34] U. Bandelow and N. Akhmediev, Phys. Rev. E 86, 026606(2012).

[35] Y. S. Tao and J. S. He, Phys. Rev. E 85, 026601(2012).

[36] Y. S. Zhang, L. J. Guo, Z. X. Zhou and J. S. He, Lett. Math. Phys., 105. 853(2015).

[37] S. H. Chen, J. M. Soto-Crespo and P. Grelu, Phys. Rev. E $90033203(2014)$.

[38] G. Mu, Z. Y. Qin, R. Grimshaw, SIAM J. Appl. Math. 75,1(2015).

[39] L. M. Ling, B. L.Guo and L. C. Zhao, Phys. Rev. E 89 041201(2014).

[40] J. S. He, L. J. Guo, Y. S. Zhang and A. Chabchoub, Proc. R. Soc. A 470, 0318 (2014).

[41] H. N. Chan, B. A. Malomed, K. W. Chow, and E. Ding, Phys. Rev. E 93, 012217(2016).

[42] Y. Zhang, J. W. Yang, K. W. Chow, and C. F. Wu, Nonlinear Anal. Real World Appl.33, 237(2017).

[43] Z. Y. Yan, V. V. Konotop, and N. Akhmediev, Phys. Rev. E 82, 036610(2010).

[44] Z. Y. Yan, Phys. Lett. A 375, 4274(2011).

[45] Z. Y. Yan, Nonlinear. Dynam. 79, 2515(2015).

[46] C. Q. Song, D. M. Xiao, Z. N. Zhu, Commun. Nonlinear Sci. Numer. Simul. 45, 13(2017).

[47] J. L. Ji, Z. N. Zhu, Commun. Nonlinear Sci. Numer. Simul. 42, 699 (2017).

[48] X. Wang, Y. Q. Li, F. Huang and Y. Chen, Commun. Nonlinear Sci. Numer. Simulat. 20, 434(2015).

[49] Alexandr Zaviyalov, Oleg Egorov, Rumen Iliew, and Falk Lederer, Phys. Rev. A 85, 013828(2012).

[50] S. H. Chen, J. M. Soto-Crespo, F. Baronio, P. Grelu, and D.Mihalache, Opt. Express 24, 15251(2016).

[51] F. Baronio, S. Wabnitz, and Y. Kodama, Phys. Rev. Lett. 116, 173901 (2016).

[52] P. H. Pioger, V. Couderc, L. Lefort, A. Barthelemy, F. Baronio, C. De Angelis, Y. Min, V. Quiring, and W. Sohler, Opt. Lett. 27, 2182 (2002).

[53] S. Tzortzakis, L. Sudrie, M. Franco, B. Prade, and M. Mysyrowicz, A. Couairon, and L. Bergé, Phys. Rev. Lett. 87, 213902 (2001).

[54] H. S. Eisenberg, R. Morandotti, Y. Silberberg, S. Bar-Ad, D. Ross, and J. S. Aitchison, Phys. Rev. Lett. 87, 043902 (2001).

[55] J. K. Ranka, R.W. Schirmer, and A. L. Gaeta, Phys. Rev. Lett. 77, 3783 (1996).

[56] X. Liu, L. J. Qian, and F.W.Wise, Phys. Rev. Lett. 82, 4631(1999).

[57] C. Klein, C. Sparber, and P. Markowich, J. Nonlinear Sci. 17, 429-470 (2007).

[58] C. Klein, J. C. Saut, J. Nonlinear Sci. 22, 763-811(2012).

[59] M. Boiti, F. Pempinelli and P. C. Sabatier, Inverse Prob. 9, 1(1993).

[60] P. C. Sabatier, Inverse Prob. 8, 263(1992).

[61] A. S. Fokas and M. J. Ablowitz, Phys. Rev. Lett. 47, 1096(1981).

[62] A. S. Fokas, Inverse Prob. 10, 19(1994).

[63] E. I. Schulman, Theor. Math. Phys. 56, 720(1984).

[64] P. M. Santini and A. S. Fokas, Commun. Math. Phys. 115, 375(1988).

[65] A. S. Fokas and P. M. Santini, Commun. Math. Phys. 116, 499(1988).

[66] G. Mu and Z. Y. Qin, Nonlinear Anal. Real World Appl. 18, 1(2014).

[67] Y. Ohta and J. K. Yang, Phys. Rev. E 86, 036604(2012).

[68] Y. Ohta and J. K. Yang, J. Phys. A: Math. Theor. 46, 105202(2013).

[69] J. C. Chen, Y. Chen, and B. F. Feng, K. Maruno Phys. Lett. A 379, 1510(2015).

[70] J. G. Rao, L. H. Wang, Y. Zhang, J.S. He, Rogue wave solutions of the Zakharov equation (accepted by Theoretical and Mathematical Physics, 2017).

[71] Y. B, Liu, A. S. Fokas, D. Mihalache, and J. S. He, Rom. Rep. Phys, 68, 1425(2016).

[72] R. Hirota, The direct method in soliton theory (Cambridge University Press, Cambridge, UK, 2004).

[73] C. Qian, J. G. Rao, Y. B. Liu, and J. S.He, Chin. Phys. Lett. 33, 110201(2016).

[74] Y. Ohta, D. S. Wang, J. K. Yang, Stud. Appl. Math., 127, 345(2011).

[75] A.S. Fokas, D.E. Pelinovsky, C. Sulaem, Physica D, 152, 189(2001). 